\title{
Error estimates for the unilateral buckling load of a plate involving the membrane efforts consistency error
}

\author{
Mekki Ayadi*,** \\ * Laboratoire de Génie Civil, Ecole Nationale d'Ingénieurs de Tunis \\ Campus universitaire, P.B.37, Le Belvédère, Tunis, Tunisie \\ ** Département d'Informatique et de Mathématiques Appliquées \\ Institut Supérieur des Sciences Appliquées et de Technologie de Sousse \\ Cité Taffala, Ibn Khaldoun, 4003 Sousse, Tunisie \\ Mekki.Ayadi@enis.rnu.tn
}

\begin{abstract}
The paper deals with error estimates for the unilateral buckling critical load of a thin plate in presence of an obstacle. The error on the membrane efforts tensor is taken into account. First, using the Mindlin's plate model together with a finite elements scheme of degree one, an error estimate, depending on the mesh size $h$, is established. In order to validate this theoretical error estimate, some numerical experiments are presented. Second, using the Kirchhoff-Love's plate model, an abstract error estimate is achieved. Its drawback is that it contains a hard term to evaluate.

RÉSUMÉ. On s'intéresse, dans ce papier, à l'estimation de l'erreur pour la charge critique de flambement unilatéral d'une plaque mince en présence d'un obstacle. L'erreur due à l'approximation de l'effort normal est prise en compte. Le choix du modèle de plaque de Mindlin et l'emploi d'un élément fini de degré un ont d'abord permis de donner une estimation de l'erreur en fonction de h. Quelques résultats numériques, ayant pour but de valider l'estimation d'erreur théorique, sont donnés. Ensuite, en utilisant le modèle de plaque de Kirchhoff-Love, une estimation d'erreur abstraite est établie. Son inconvénient est qu'elle contient un terme difficile à évaluer en fonction de $h$.

KEYWORDS: unilateral buckling, buckling critical load, finite element method, error estimate, consistency error.

MOTS-CLÉS : flambement unilatéral, charge critique de flambement, méthode d'éléments finis, estimation d'erreur, erreur de consistance.
\end{abstract}

DOI:10.3166/REMN.17.1003-1038 @ 2008 Lavoisier, Paris 


\section{Introduction}

Consider a thin plate of thickness $2 \varepsilon$ occupying a two-dimensional bounded open set $\omega$. Assume that it is supported on the whole of its edge $\gamma$, clamped on a part $\gamma_{0}$ of its edge whose Lebesgue measure is not zero, and simply supported on $\gamma / \gamma_{0}$. Furthermore, the plate is subjected to a one-parameter plane compressive load $\lambda . g$ on another part $\gamma_{1}$ of its edge, see Figure 1.

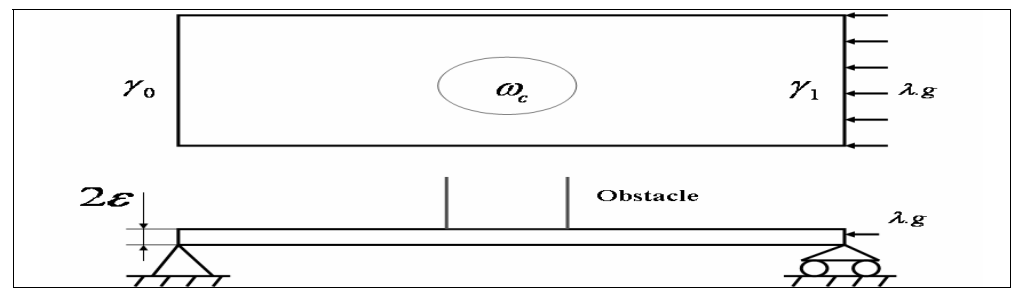

Figure 1. A rectangular plate is in presence of an obstacle

The plate is in presence of a rigid fixed plane obstacle that lies just above it, see Figure 1. The contact between the plate and the obstacle is supposed to be without friction. If the obstacle is not initially in contact with the plate, the problem becomes classical in the sense that it has the same solution as the linear one.

The unilateral buckling and even the unilateral post-buckling of thin plates have been tackled and investigated by many authors since the late seventies. Let us cite, in particular, (Do, 1975, 1976; Riddell, 1977; Cimetière, 1980, 1985; Kucera, 1982; Quittner, 1986; Goeleven et al., 1995; Shahwan et al., 1998; Goeleven et al., 1998; Smith et al., 1999, 2000; Bradford et al., 2000; Chai, 2002; Febres et al., 2003; Shen et al., 2004; Parry et al., 2005; Mouradova et al., 2006; Ayadi, 2006; Ma et al., 2007). Recently, a new contribution to unilateral buckling problem has been brought by Ayadi; he succeeded to establish error estimates for the unilateral buckling critical load of a thin plate in presence of an obstacle if the error on the membrane efforts tensor is neglected (Ayadi, 2007).

In this paper, we will deal with error estimates for the unilateral buckling critical load when the consistency error on the membrane efforts tensor is taken into account.

The layout of this paper is as follows. The first section is devoted to the description of the unilateral buckling model. In the second section, the adequate framework so that the critical state of unilateral buckling exists, see (Do, 1975, 1976; Riddell, 1977), is first briefly recalled. Then, in order to motivate the interest we are manifesting for the unilateral buckling of a thin plate, a delaminated multilayered plate is considered and the critical load as well as the corresponding buckling mode are computed in the two cases: without and with unilateral contact between the two lips of the crack. The third 
Section is devoted to an error estimate for the pure membrane problem. In the fourth Section, using the Mindlin plate theory together with a conformal finite elements scheme of degree one (triangle with three nodes), an error estimate for the unilateral buckling critical load, taking into account the error on the membrane efforts tensor, depending on the mesh size $\mathrm{h}$ is achieved. In order to numerically validate this error formula, a numerical experiment, where the plate and the obstacle are both rectangular, is handled. Three curves are plotted in the same figure. Two curves give the dependence of the buckling critical load, in the absence and in the presence of the obstacle, upon the mesh size. The third curve gives the dependence of the difference of the two buckling critical loads (without and with contact) upon the mesh size. The latter is, following the theoretical error estimate, expected to be linear. The numerical results obtained confirm with the theoretical error estimate for the unilateral critical load when the error on the membrane efforts tensor is not neglected. In the fifth Section, using the Kirchhoff-Love plate theory and a conformal finite elements scheme, an abstract error estimate for the unilateral buckling critical load, taking into account the error on the membrane efforts tensor, is established. Its drawback is that it contains a term very hard to evaluate.

\section{Mathematical modeling of unilateral buckling}

When taking into account the unilateral contact condition and considering a nonlinear elastic constitutive law, we obtain a very difficult mathematical problem (see (Ciarlet, 1986; Ciarlet et al., 1977; Duvaut et al., 1972). Nevertheless, we know a particular solution $u^{p}$ to the latter. It is the linear elasticity solution, obtained with linearized strains, for which the vertical displacement is zero (there is not bending) and the plane displacements are solution to the following variational equation:

$$
\sum_{\alpha, \beta, \nu, \mu=1}^{2} \int_{\omega} E_{\alpha \beta \nu \mu} \frac{\partial u_{\nu}^{p}}{\partial x_{\mu}} \frac{\partial v_{\alpha}}{\partial x_{\beta}} d \omega=\lambda \sum_{\alpha=1}^{2} \int_{\gamma_{1}} G_{\alpha} v_{\alpha} d \gamma \forall v \in V
$$

Where $\left(E_{\alpha \beta \nu \mu}\right)$ is the membrane stiffness tensor for the linear elastic constitutive law (depending on Young's modulus and the Poisson's ratio),

$$
G_{\alpha}=\int_{-\varepsilon}^{\varepsilon} g_{\alpha}\left(x_{1}, x_{2}, x_{3}\right) d x_{3}, \alpha \in\{1,2\}
$$

$g_{1}$ and $g_{2}$ are the components of the plane compressive load $g$ (see Figure 1), and

$$
V=\left\{v \in\left(H^{1}(\omega)\right)^{2}: v=0 \text { on } \gamma_{0}\right\}
$$


Problem [1] is so a pure membrane type.

The space $V$ is equipped with the following norm:

$$
\forall v \in V,\|v\|_{V}=\left(\left\|v_{1}\right\|_{1, \omega}^{2}+\left\|v_{2}\right\|_{1, \omega}^{2}\right)^{\frac{1}{2}}
$$

The following usual assumptions are made.

(i) The boundary $\gamma$ of the bounded open set $\omega$ is supposed to be smooth enough,

(ii) $g_{1}, g_{2} \in L^{2}\left(\gamma_{1} \times\right]-\varepsilon, \varepsilon[)$ so that $G_{\alpha} \in L^{2}\left(\gamma_{1}\right), \alpha \in\{1,2\}$,

(iii) $E_{\alpha \beta \nu \mu} \in L^{\infty}(\omega)$ for all $(\alpha, \beta, \nu, \mu) \in\{1,2\}^{4}$,

(iv) $\exists k>0 ; \forall \theta \in \Theta_{s, 2}: \sum_{\alpha, \beta, \nu, \mu=1}^{2} E_{\alpha \beta \nu \mu} \theta_{\alpha \beta} \theta_{\nu \mu} \geq k \sum_{\alpha, \beta=1}^{2}\left(\theta_{\alpha \beta}\right)^{2}$,

where $\Theta_{s, 2}$ denotes the set of all symmetric tensors of order two: that is the ellipticity property. It is shown in (Ciarlet, 1986) and in (Duvaut et al., 1972) that, under the assumptions (i) - (iv) above, problem [1] admits a unique solution $u^{p} \equiv u^{p}(\lambda)=\lambda u^{g}$, where $u^{g}$ is given in $V$ ( $u^{g}$ does not depend of $\left.\lambda\right)$. Moreover, the tensor of membrane efforts is (see for instance (Ayadi et al., 1990; Ayadi, 1993; Ciarlet et al., 1977) expressed by:

$$
n_{\alpha \beta}^{p}=\sum_{\nu, \mu=1}^{2} E_{\alpha \beta \nu \mu} \frac{\partial u_{\nu}^{p}}{\partial x_{\mu}}=\lambda n_{\alpha \beta}^{g}, \quad \alpha, \beta \in\{1,2\} .
$$

Looking for a non-trivial solution to the nonlinear problem described above, we need the linearizing technique: set $u=u^{p}+w$ and show that the deflection $w_{3}$ of the plate is solution to the following inequality:

$$
\sum_{\alpha, \beta, \nu, \mu=1}^{2} \int_{\omega} D_{\alpha \beta \nu \mu} \frac{\partial^{2} w_{3}}{\partial x_{\nu} \partial x_{\mu}} \frac{\partial^{2}\left(v-w_{3}\right)}{\partial x_{\alpha} \partial x_{\beta}} d \omega \geq \lambda \sum_{\alpha, \beta=1}^{2} \int_{\omega} n_{\alpha \beta}^{g} \frac{\partial w_{3}}{\partial x_{\alpha}} \frac{\partial\left(v-w_{3}\right)}{\partial x_{\beta}} d \omega
$$

for all admissible deflection $v$, where $\left(D_{\alpha \beta \nu \mu}\right)$ denotes the bending rigidity tensor of the plate. The other components $w_{1}$ and $w_{2}$, of the displacement $w$, are related to the deflection $w_{3}$ by Kirchhoff-Love formulae (Ciarlet et al., 1977). 


\section{Mathematical framework and existence results}

Let us start by defining the adequate framework used in this paper so that problem [3] admits at least one solution.

$$
\begin{gathered}
H=\left\{v \in H^{1}(\omega): v=0 \text { on } \gamma_{0}\right\}, \\
W=\left\{v \in H^{2}(\omega): v=\frac{\partial v}{\partial n}=0 \text { on } \gamma_{0}\right\}, \\
K=\left\{v \in W: v \leq 0 \text { in } \omega_{c}\right\},
\end{gathered}
$$

$\omega_{c}$ being a subset of $\omega$ where the contact between the obstacle and the plate would occur, and $\partial v / \partial n$ is the derivative of the function $v$ with respect to the outward unit vector normal $n$ to the boundary $\gamma$ of $\omega$.

The Sobolev spaces $H$ and $W$ are respectively equipped with the following norms:

$$
|u|_{1, \omega}=\left(\int_{\omega} \sum_{\alpha=1}^{2}\left(\frac{\partial u}{\partial x_{\alpha}}\right)^{2}\right)^{\frac{1}{2}},\|u\|_{2, \omega}=\left(\int_{\omega} \sum_{|\alpha| \leq 2}\left(\partial^{\alpha} u\right)^{2}\right)^{\frac{1}{2}} .
$$

Let $a: W \times W \rightarrow I R$ and $b\left(n^{g}\right): H \times H \rightarrow I R$ be the bilinear forms defined by

$$
\begin{gathered}
a(u, v)=\sum_{\alpha, \beta, \nu, \mu=1}^{2} \int_{\omega} D_{\alpha \beta \nu \mu} \frac{\partial^{2} u}{\partial x_{\nu} \partial x_{\mu}} \frac{\partial^{2} v}{\partial x_{\alpha} \partial x_{\beta}} d \omega, \\
b\left(n^{g}\right)(u, v)=\sum_{\alpha, \beta=1}^{2} \int_{\omega} n_{\alpha \beta}^{g} \frac{\partial u}{\partial x_{\alpha}} \frac{\partial v}{\partial x_{\beta}} d \omega .
\end{gathered}
$$

The bilinear forms $b\left(n^{g}\right)$ and $a$ are obviously continuous in the spaces $H$ and $W$ respectively. Therefore, there exist two positive constants $M$ and $N\left(n^{g}\right)$ such that

$$
\begin{gathered}
|a(u, v)| \leq M\|u\|_{2, \omega}\|v\|_{2, \omega}, \forall u, v \in W, \\
\left|b\left(n^{g}\right)(u, v)\right| \leq N\left(n^{g}\right)|u|_{1, \omega}|v|_{1, \omega}, \forall u, v \in H .
\end{gathered}
$$


Assume now that the bending rigidity tensor $\left(D_{\alpha \beta \nu \mu}\right)$ satisfies the ellipticity property (iv) so that the bilinear form $a$ is $W$-elliptic (see (Ciarlet et al., 1977), (Destuynder, 1990), (Duvaut et al., 1972)). That means there exists a positive constant $\alpha$ such that

$$
a(v, v) \geq \alpha\|v\|_{2, \omega}^{2}, \forall v \in W
$$

Within the framework defined above, problem [3] is mathematically well posed as stated by the following theorem.

Theorem 3.1. There exist $\lambda_{1}>0$ and nonzero vector $w_{3} \in K$ such that

$$
a\left(w_{3}, v-w_{3}\right) \geq \lambda_{1} b\left(n^{g}\right)\left(w_{3}, v-w_{3}\right), \forall v \in K
$$

Moreover, $\lambda_{1}$ is the minimum of the Rayleigh quotient over the closed convex cone $K$, which is realized on $w_{3}$ :

$$
\lambda_{1}=\min _{v \in K-\{0\}} \frac{a(v, v)}{b\left(n^{g}\right)(v, v)} .
$$

Proof: See (Riddell, 1977).

After stating that the unilateral critical state $\left(\lambda_{1}, w_{3}\right)$ exists and before starting the main aim of the paper: namely error estimates for the unilateral critical load of a thin plate involving the membrane efforts consistency error, let us give an interesting application of unilateral buckling. That is the buckling of a delaminated multilayered plate (Ayadi et al., 1990). The crack is situated between two fiber layers, see Figure 2. The plate is modeled as the union of three plates: the healthy part $\Omega_{1}$, the upper delaminated part $\Omega_{2}$ and the lower delaminated part $\Omega_{3}$. The three plates are connected with each other by ensuring the continuity of the deflection and the mid plan rotations in the bottom of the crack (Nevers, 1986). The crack can be exenterated from the mid plan of the plate. Under a certain compression loading, the thinnest lip of the crack, at least, is expected to buckle; that is the local buckling. In order to avoid the material interpenetration, a contact condition between the two lips $\Omega_{2}$ and $\Omega_{3}$ of the crack must be imposed.

The Mindlin plate theory is used because it only requires a continuous finite element. Some numerical experiments, obtained in the case of a homogenous and isotropic material, are here after presented. 


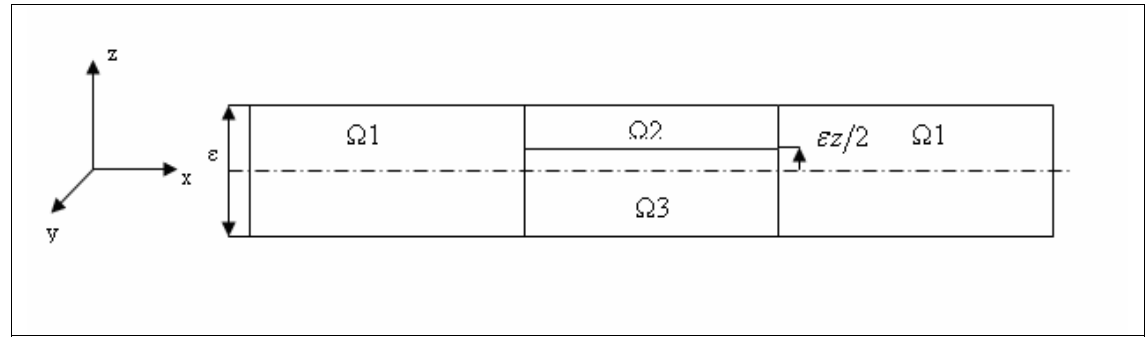

Figure 2. Transversal cross section of a delaminated plate, $z \in]-1,1[$

Consider the following plate which is, for the moment, considered healthy.

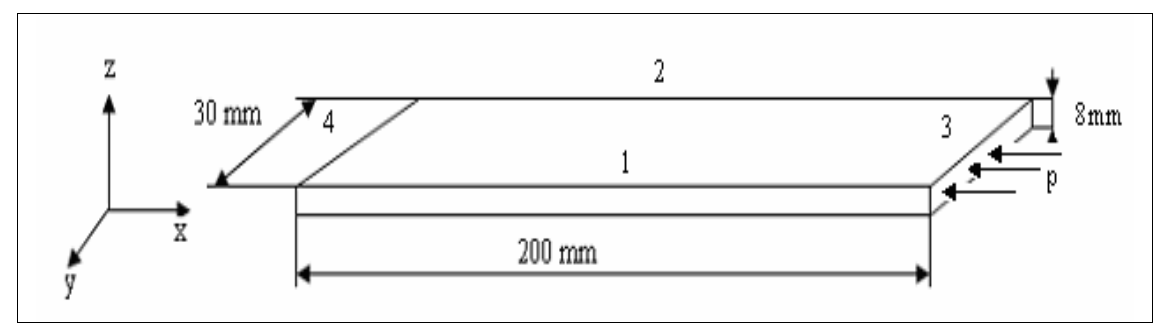

Figure 3. A healthy plate

The boundary conditions are: the plane displacement along the $x$ axis is fixed null on the edge (4); the deflection is fixed null on the whole edge of the plate (Simply supported plate); on the edge (3), a uniform compression loading $\mathrm{p}$ is applied.

The mechanical futures are: the Young's modulus $E=10^{9} \mathrm{~Pa}$ and the Poisson's coefficient $\nu=0.3$.

The following Figure 4 shows the healthy plate mesh. There are 736 finite triangles with three nodes, 421 nodes and 1263 degrees of freedom.

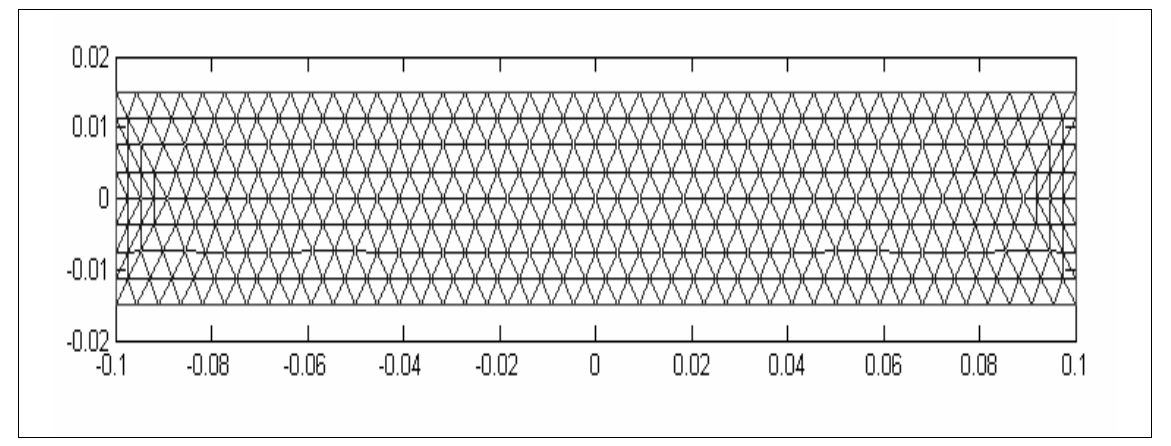

Figure 4. A healthy plate mesh 
The following Figure 5 shows the buckling mode of the above plate corresponding to the numerical critical load: $\varepsilon p_{\text {healthy }}^{\text {crit }}=1.866310^{6} \mathrm{~N} / \mathrm{m}$.

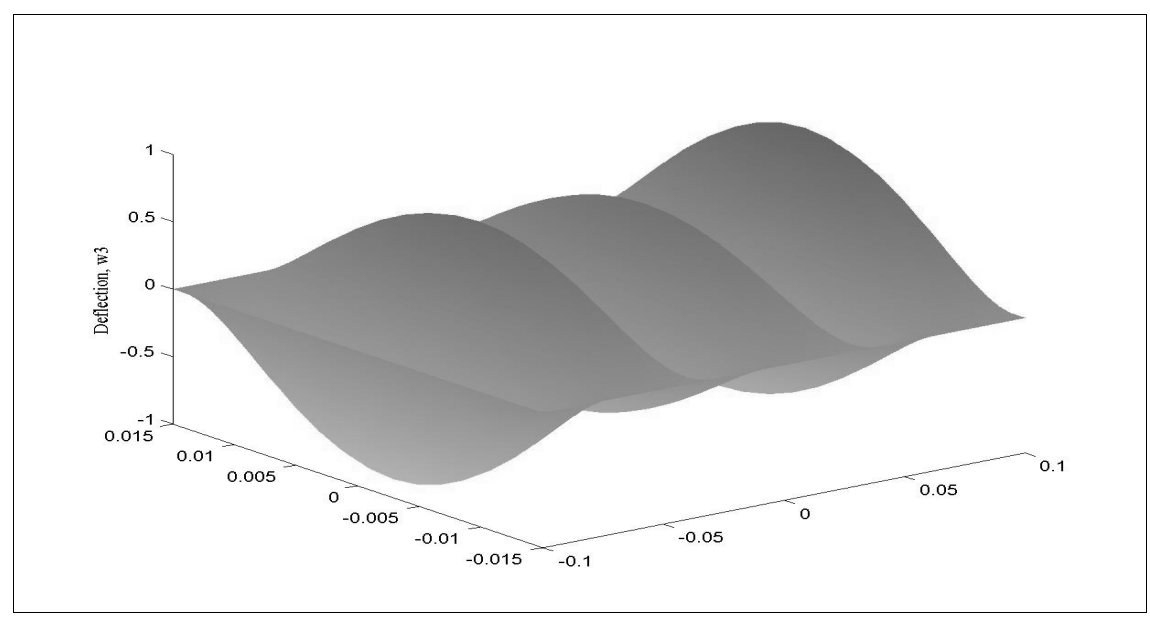

Figure 5. The buckling mode of the healthy plate

Suppose now that the plate of Figure 3 is delaminated in the following way $(z=0.5)$.

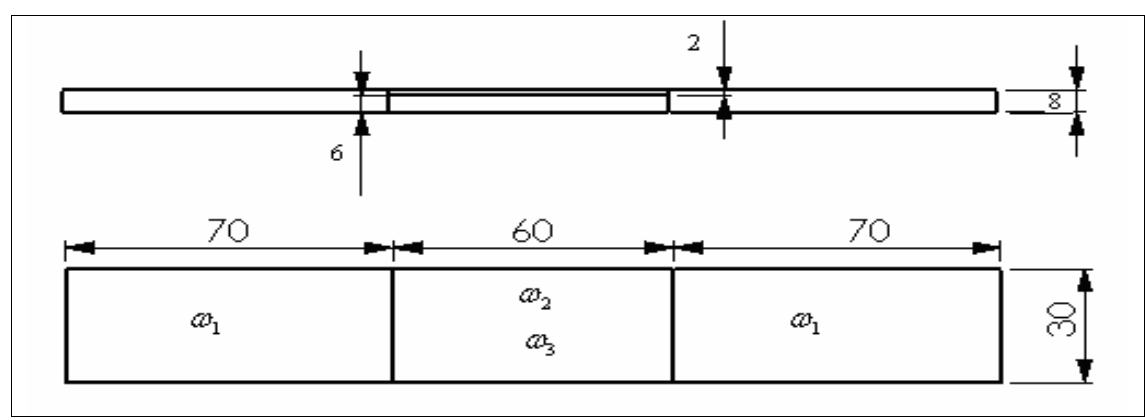

Figure 6. The delaminated plate

The same boundary conditions as the healthy plate of Figure 3 are imposed. The following Figure 7 shows the healthy part mesh (mesh of $\omega_{1}$ ) of the delaminated plate. There are 576 finite triangles with three nodes, 338 nodes and 1014 degrees of freedom. 


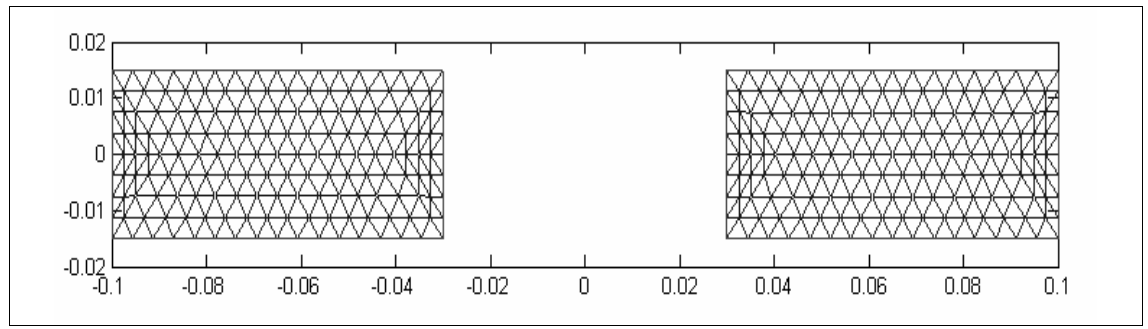

Figure 7. The healthy part mesh

The two delaminated part have the same mesh because $\omega_{2}=\omega_{3}$, see the following Figure 8. There are 256 finite triangles with three nodes and 153 nodes, which give 459 degrees of freedom in each delaminated part.

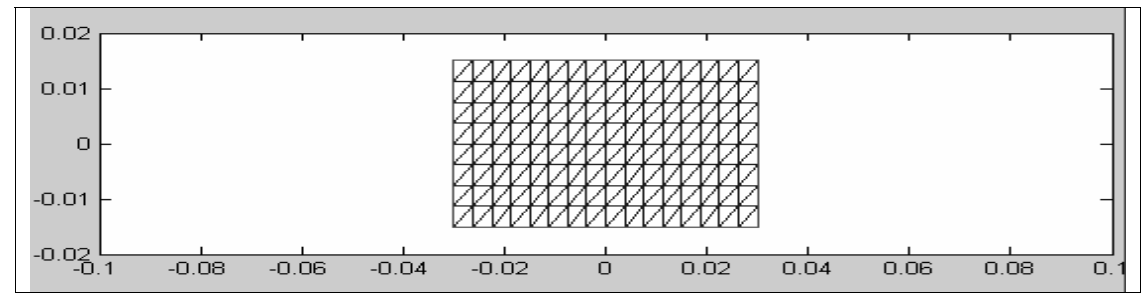

Figure 8. The delaminated part mesh

If the unilateral contact is not taken into account, we observe an important interpenetration of the crack lips. Only the upper delaminated part buckles as exemplify Figures 9 and 10. The numerical critical load is: $\varepsilon p_{\text {without contact }}^{\text {crit }}=3.853210^{5} \mathrm{~N} / \mathrm{m}$.

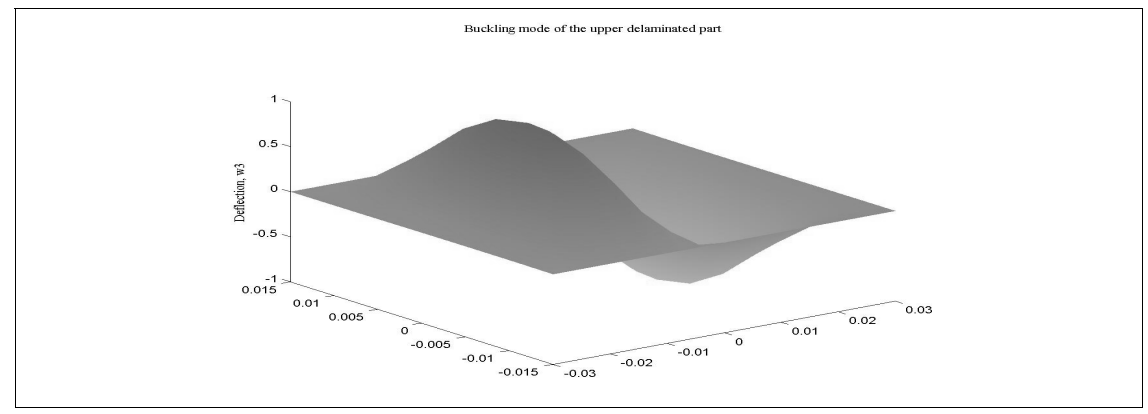

Figure 9. The buckling mode of the upper delaminated part without contact 


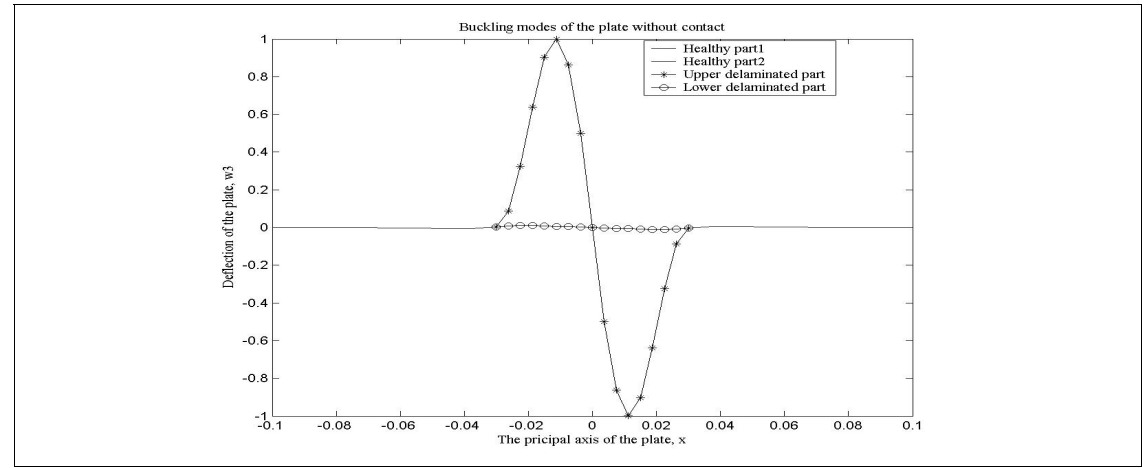

Figure 10. Cross section a long the $x$ axis $(y=0)$ of the buckling mode of the whole plate

If we take into account the unilateral contact (the interpenetration of the upper lip and the lower lip of the crack is prohibited) by imposing the condition: the upper deflection $w_{3}^{2}\left(a_{i}\right)$ is great or equal to the lower deflection $w_{3}^{3}\left(a_{i}\right)$ for all mesh node $a_{i}$ of the delaminated part, we observe that the healthy part does not buckle and does not even bend. The lower delaminated part - at the opposite - slightly bends (a deflection of $\operatorname{order} \varepsilon$ ), which is due to the buckling of the upper delaminated part, see Figures 12 and 13. The numerical critical load is: $\varepsilon p_{\text {with contact }}^{\text {crit }}=4.150510^{5} \mathrm{~N} / \mathrm{m}$. For more details, see (Shiri et al., 2007).

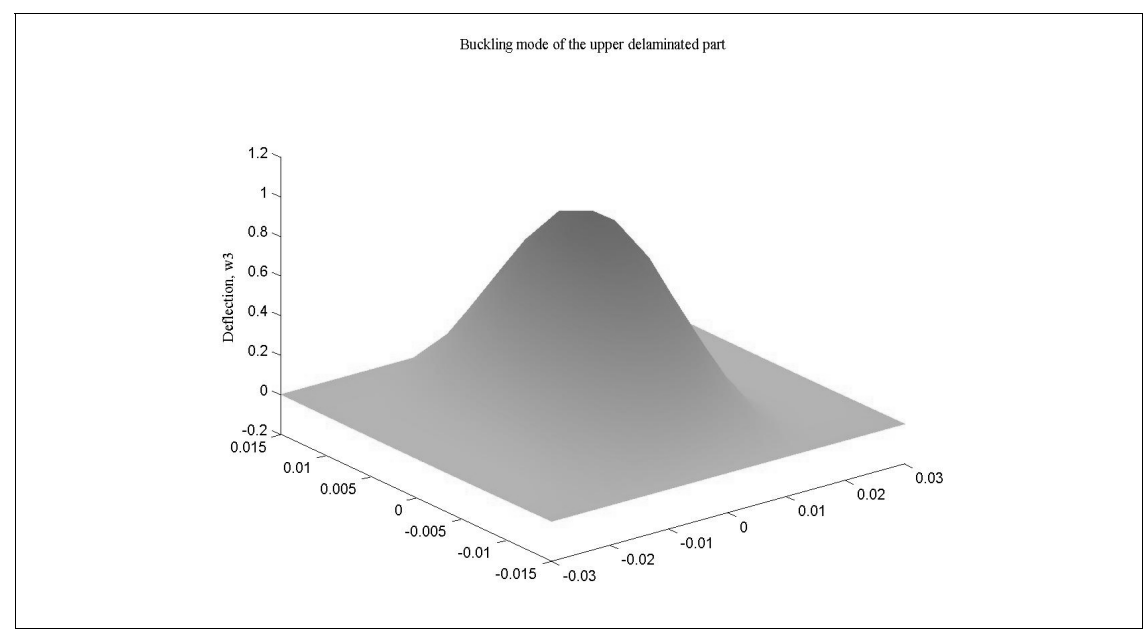

Figure 11. The buckling mode of the upper delaminated part with contact 


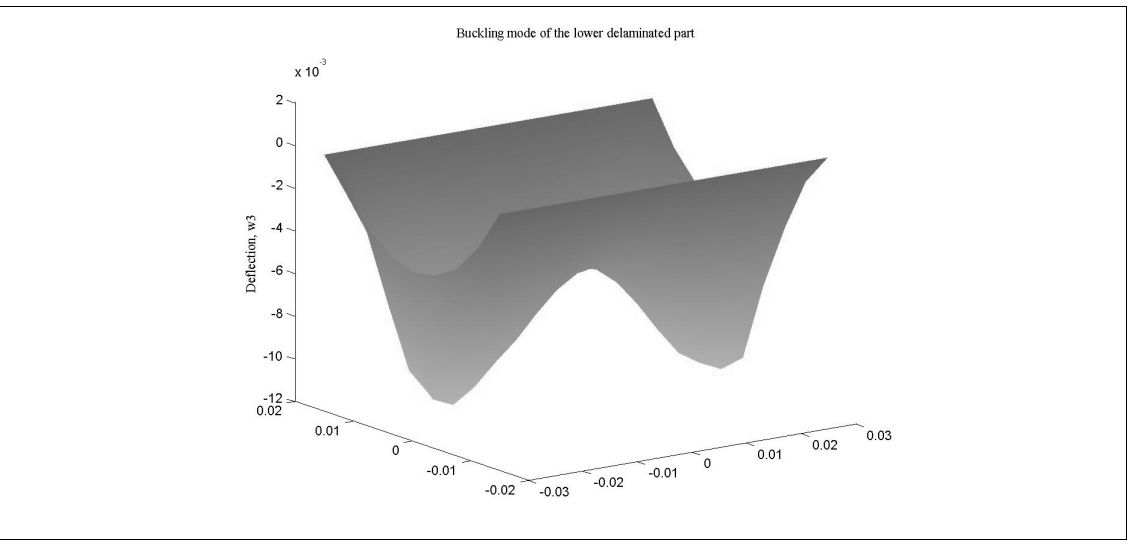

Figure 12. The buckling mode of the lower delaminated part with contact

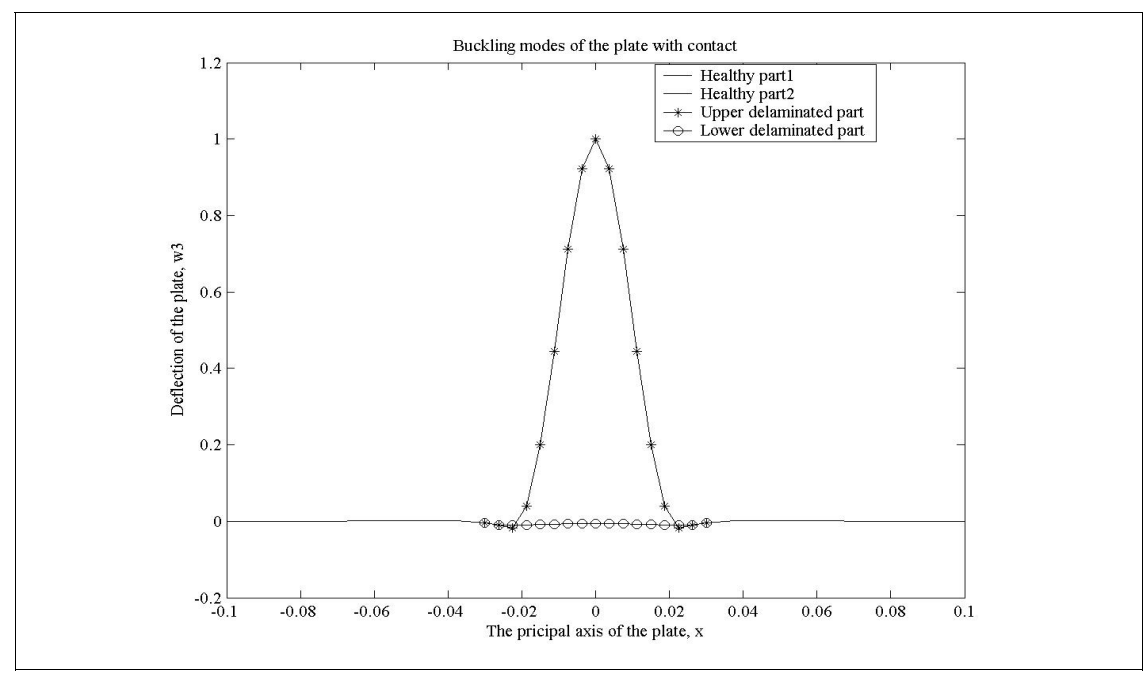

Figure 13. Transversal section a long the $x$ axis $(y=0)$ of the buckling mode with contact

As a conclusion, the use of Mindlin's plate theory and the triangle with three nodes has allowed to construct a numerical model for unilateral buckling of delaminated plates whose plane crack can be exenterated from the mid plan. This model performs the computing of the critical state: the buckling critical load and the corresponding buckling mode. The numerical results presented above show that the presence of delaminations in a multilayered plate enormously weakens it. Indeed, we have: 


$$
\frac{\varepsilon p_{\text {healthy }}^{\text {crit }}}{\varepsilon p_{\text {without contact }}^{\text {crit }}}=4.8435 \text {. }
$$

The plate being delaminated, taking into account the unilateral contact in the delaminations region strengthens it:

$$
\frac{\varepsilon p_{\text {with contact }}^{\text {crit }}}{\varepsilon p_{\text {without contact }}^{\text {crit }}}=0.9284
$$

\section{Error estimates for the membrane efforts}

First, we will deal with the pure membrane problem [1] (there is not bending). The reader is reminded that the solution $u^{p}$ of problem [1] is also a particular solution to the more difficult problem of buckling of the plate in presence of an obstacle.

Let $\left(\mathscr{J}_{h}\right)$ be a regular triangulation of $\bar{\omega}$. Then, the space $V$ is approximated by the finite-dimensional subspace

$$
V_{h}=\left\{v_{h} \in\left(C^{0}(\bar{\omega})\right)^{2}: v_{h i \mid T} \in P_{k}(T), k=1 \text { or } 2, T \in\left(\mathscr{J}_{h}\right) \text { and } v_{h}=0 \text { on } \gamma_{0}\right\},
$$

where

$$
\begin{gathered}
\mathscr{P}_{1}(T)=\left\{P_{1}=a_{00}+a_{10} X+a_{01} Y: a_{i j} \in I R\right\} \\
\mathscr{P}_{2}(T)=\left\{P_{1}=a_{00}+a_{10} X+a_{01} Y+a_{11} X Y+a_{20} X^{2}+a_{02} Y^{2}: a_{i j} \in I R\right\}
\end{gathered}
$$

are respectively the spaces of polynomial functions of degree less or equal to one and of degree less or equal to two. Then, problem [1] is approximated by: find $u_{h}^{p} \in V_{h}$ such that

$$
\sum_{\alpha, \beta, \nu, \mu=1}^{2} \int_{\omega} E_{\alpha \beta \nu \mu} \frac{\partial u_{h \nu}^{p}}{\partial x_{\mu}} \frac{\partial v_{h \alpha}}{\partial x_{\beta}} d \omega=\lambda \sum_{\alpha=1}^{2} \int_{\gamma_{1}} G_{\alpha} v_{h \alpha} d \gamma, \forall v_{h} \in V_{h}
$$

Under the same assumptions (i)-(iv) that make problem [1] admit a unique solution $u^{p}=\lambda u^{g} \in V$, the discrete problem [8] admits also a unique solution $u_{h}^{p}=\lambda u_{h}^{g} \in V_{h}\left(u_{h}^{g}\right.$ does not depend of $\left.\lambda\right)$ whose correspondent membrane efforts tensor is 


$$
n_{h \alpha \beta}^{p}=\sum_{\nu, \mu=1}^{2} E_{\alpha \beta \nu \mu} \frac{\partial u_{h \nu}^{p}}{\partial x_{\mu}}=\lambda \cdot n_{h \alpha \beta}^{g}, \quad \alpha, \beta \in\{1,2\}
$$

Furthermore, let

$$
|v|_{k+1, \omega}=\left(\left|v_{1}\right|_{k+1, \omega}^{2}+\left|v_{2}\right|_{k+1, \omega}^{2}\right)^{\frac{1}{2}} \text { where }\left|v_{i}\right|_{k+1, \omega}^{2}=\sum_{i_{1}+i_{2}=k+1}\left\|\frac{\partial^{k+1} v_{i}}{\partial x_{1}^{i_{1}} \partial x_{2}^{i_{2}}}\right\|_{0, \omega}^{2}, i=1,2,
$$

be the semi norm of the Sobolev space $\left(H^{k+1}(\omega)\right)^{2}$, we have the following error estimates.

Theorem 4.1. Suppose that

$$
u^{g} \in\left(H^{k+1}(\omega)\right)^{2} .
$$

Then, there exists a positive constant $c_{1}(\omega)$ independent of the subspace $V_{h}$ such that

$$
\left\|u^{g}-u_{h}^{g}\right\|_{V} \leq c_{1}(\omega) h^{k}\left|u^{g}\right|_{k+1, \omega} .
$$

Proof: See for instance (Raviart et al., 1983).

Corollary 4.2. Suppose that

$$
u^{g} \in\left(H^{k+1}(\omega)\right)^{2} .
$$

Then, there exists a positive constant $c_{2}(\omega)$ independent of the subspace $V_{h}$ such that

$$
\max _{1 \leq \alpha, \beta \leq 2}\left\|\left(n^{g}-n_{h}^{g}\right)_{\alpha \beta}\right\|_{0, \omega} \leq c_{2}(\omega) h^{k}\left|u^{g}\right|_{k+1, \omega} .
$$

Proof: Let $\alpha, \beta$ be a pair of integers such that $1 \leq \alpha, \beta \leq 2$. According to the hypothesis (iii), the following quantity exists.

$$
E_{\alpha \beta}=\max _{1 \leq \nu, \mu \leq 2}\left\|E_{\alpha \beta \nu \mu}\right\|_{L^{\infty}(\omega)} .
$$

Then, formulae [2] and [9] involve

$$
\left|\left(n_{h}^{g}-n^{g}\right)_{\alpha \beta}\right| \leq E_{\alpha \beta}\left\{\left|\left(u_{h}^{g}-u^{g}\right)_{1,1}\right|+\left|\left(u_{h}^{g}-u^{g}\right)_{1,2}\right|+\left|\left(u_{h}^{g}-u^{g}\right)_{2,1}\right|+\left|\left(u_{h}^{g}-u^{g}\right)_{2,2}\right|\right\} \text {. }
$$


Taking the square of the last inequality, we obtain

$$
\begin{aligned}
\left|\left(n_{h}^{g}-n^{g}\right)_{\alpha \beta}\right|^{2} \leq\left. E_{\alpha \beta}^{2}\left\{\left|\left(u_{h}^{g}-u^{g}\right)_{1,1}\right|^{2}+\left|\left(u_{h}^{g}-u^{g}\right)_{1,2}\right|^{2}+\left|\left(u_{h}^{g}-u^{g}\right)_{2,1}\right|^{2}+|| u_{h}^{g}-u^{g}\right)_{2,2}\right|^{2}+ \\
2\left|\left(u_{h}^{g}-u^{g}\right)_{1,1}\right|\left|\left(u_{h}^{g}-u^{g}\right)_{1,2}\right|+2\left|\left(u_{h}^{g}-u^{g}\right)_{1,1}\right|||\left(u_{h}^{g}-u^{g}\right)_{2,1} \mid+ \\
2\left|\left(u_{h}^{g}-u^{g}\right)_{1,1}\right|\left|\left(u_{h}^{g}-u^{g}\right)_{2,2}\right|+2\left|\left(u_{h}^{g}-u^{g}\right)_{1,2}\right|\left|\left(u_{h}^{g}-u^{g}\right)_{2,1}\right|+ \\
\left.2\left|\left(u_{h}^{g}-u^{g}\right)_{1,2}\right|\left|\left(u_{h}^{g}-u^{g}\right)_{2,2}\right|+2\left|\left(u_{h}^{g}-u^{g}\right)_{2,1}\right|\left|\left(u_{h}^{g}-u^{g}\right)_{2,2}\right|\right\} .
\end{aligned}
$$

This, by using the identity $2 a b \leq a^{2}+b^{2}$, yields

$$
\left|\left(n_{h}^{g}-n^{g}\right)_{\alpha \beta}\right|^{2} \leq 4 E_{\alpha \beta}^{2}\left\{\left|\left(u_{h}^{g}-u^{g}\right)_{1,1}\right|^{2}+\left|\left(u_{h}^{g}-u^{g}\right)_{1,2}\right|^{2}+\left|\left(u_{h}^{g}-u^{g}\right)_{2,1}\right|^{2}+\left|\left(u_{h}^{g}-u^{g}\right)_{2,2}\right|^{2}\right\}
$$

Taking then the integral of the above inequality gives

$$
\begin{aligned}
\left\|\left(n_{h}^{g}-n^{g}\right)_{\alpha \beta}\right\|_{0, \omega}^{2} & \leq 4 E_{\alpha \beta}^{2}\left\{\left|\left(u_{h}^{g}-u^{g}\right)_{1}\right|_{1, \omega}^{2}+\|\left.\left(u_{h}^{g}-u^{g}\right)_{2}\right|_{1, \omega} ^{2}\right\} \\
& \leq 4 E_{\alpha \beta}^{2}\left\{\left\|\left(u_{h}^{g}-u^{g}\right)_{1}\right\|_{1, \omega}^{2}+\left\|\left(u_{h}^{g}-u^{g}\right)_{2}\right\|_{1, \omega}^{2}\right\} \\
& =4 E_{\alpha \beta}^{2}\left\|u_{h}^{g}-u^{g}\right\|_{V}^{2}
\end{aligned}
$$

Set

$$
E_{\max }=\max _{1 \leq \alpha, \beta \leq 2} E_{\alpha \beta}
$$

According to [10], we obtain the inequality [11] with $c_{2}(\omega)=2 c_{1}(\omega) E_{\max }$.

Let now $b\left(n_{h}^{g}\right): H \times H \rightarrow I R$ be the family of bilinear forms defined by

$$
b\left(n_{h}^{g}\right)(u, v)=\sum_{\alpha, \beta=1}^{2} \int_{\omega} n_{h \alpha \beta}^{g} \frac{\partial u}{\partial x_{\alpha}} \frac{\partial v}{\partial x_{\beta}} d \omega
$$

Then, we have the very interesting following inequality. 
Corollary 4.3. Suppose that

$$
u^{g} \in\left(H^{k+1}(\omega)\right)^{2} .
$$

Then, there exists a positive constant $c_{3}(\omega)$ independent of the subspace $V_{h}$ such that

$$
\left|b\left(n_{h}^{g}\right)(v, v)-b\left(n^{g}\right)(v, v)\right| \leq c_{3}(\omega) h^{k}\left|u^{g}\right|_{k+1, \omega}\|v\|_{2, \omega}^{2}, \forall v \in H^{2}(\omega) .
$$

Proof: $\omega$ being a bounded open set of $I R^{2}$ that is supposed to be smooth enough (hypothesis (i)). Following the very interesting Rellich-Kondrachov theorem, namely the imbedding of the space $H^{1}(\omega)$ in the space $L^{q}(\omega)$ is compact for all real number $q \geq 1$ (Brézis, 1983), we particularly have:

$$
\forall v \in H^{1}(\omega),\|v\|_{L^{4}(\omega)} \leq C\|v\|_{1, \omega} .
$$

Let $v \in H^{2}(\omega)$, according to the Hölder inequality (Brézis, 1983) and [13], we have

$$
\begin{aligned}
\left|b\left(n_{h}^{g}\right)(v, v)-b\left(n^{g}\right)(v, v)\right| & \leq \sum_{\alpha, \beta=1}^{2}\left\|\left(n_{h}^{g}-n^{g}\right)_{\alpha \beta}\right\|_{0, \omega}\left\|\frac{\partial v}{\partial x_{\alpha}}\right\|_{L^{4}(\omega)}\left\|\frac{\partial v}{\partial x_{\beta}}\right\|_{L^{4}(\omega)} \|\left(\max _{1 \leq \alpha, \beta \leq 2}\left\|\left(n_{h}^{g}-n^{g}\right)_{\alpha \beta}\right\|_{0, \omega} \sum_{\alpha, \beta=1}^{2}\left\|\frac{\partial v}{\partial x_{\alpha}}\right\|_{L^{4}(\omega)}\left\|\frac{\partial v}{\partial x_{\beta}}\right\|_{L^{4}(\omega)}\right. \\
& \left.\leq 2 \max _{1 \leq \alpha, \beta \leq 2}\left\|\left(n_{h}^{g}-n^{g}\right)_{\alpha \beta}\right\|_{0, \omega}\|\| \frac{\partial v}{\partial x_{1}}\left\|_{L^{4}(\omega)}^{2}+\right\| \frac{\partial v}{\partial x_{2}} \|_{L^{4}(\omega)}^{2}\right) \\
& \leq 2 C^{2} \max _{1 \leq \alpha, \beta \leq 2}\left\|\left(n_{h}^{g}-n^{g}\right)_{\alpha \beta}\right\|_{0, \omega}\left(\left\|\frac{\partial v}{\partial x_{1}}\right\|_{1, \omega}^{2}+\left\|\frac{\partial v}{\partial x_{2}}\right\|_{1, \omega}^{2}\right) \\
& \leq 2 C^{2} \max _{1 \leq \alpha, \beta \leq 2}\left\|\left(n_{h}^{g}-n^{g}\right)_{\alpha \beta}\right\|\|\| v \|_{0, \omega}^{2},
\end{aligned}
$$

which, using inequality [11], yields inequality [12] with $c_{3}(\omega)=4 c_{1}(\omega) E_{\max } C^{2}$.

Suppose now that the membrane efforts tensor $\left(n_{\alpha \beta}^{g}\right)$ verifies the ellipticity property (which is the case of a compression loading and several kinds of boundary conditions): there exists a positive constant $\eta>0$ such that 


$$
\sum_{\alpha, \beta=1}^{2} n_{\alpha \beta}^{g} \theta_{\alpha} \theta_{\beta} \geq \eta \sum_{\alpha=1}^{2} \theta_{\alpha}^{2}, \forall \theta \in I R^{2}
$$

Then, we can easily deduce that

$$
b\left(n^{g}\right)(v, v) \geq \eta|v|_{1, \omega}^{2}, \forall v \in H
$$

Therefore, the embedding of the space $W$ in the space $H$, supposed to be equipped with the norm $|\cdot|_{b}: H \rightarrow I R_{+}: v \mapsto|v|_{b}=\sqrt{b\left(n^{g}\right)(v, v)}$, is continuous:

$$
|v|_{b} \leq \sqrt{N\left(n^{g}\right)}\|v\|_{2, \omega}, \forall v \in W
$$

\section{Mindlin's plate model}

\subsection{Error estimates for the buckling critical load}

Now, we will deal with the pure bending problem [3] (there is not a membrane effect). In order to minimize the number of degrees of freedom, the Kirchhoff-Love's plate model is often replaced by the Mindlin's one. A comparison between the two models exists in (Davet et al., 1985). The Mindlin's plate model involves, as unknowns, the deflection $w_{3}$ and the two rotations $\theta_{1}$ and $\theta_{2}$ of the mid plan of the plate which are related by the following formulae (Ciarlet et al., 1977; Destuynder, 1990):

$$
\theta_{\alpha}=-\frac{\partial w_{3}}{\partial x_{\alpha}}, \alpha=1,2
$$

The changing of the plate model obviously involves a changing of the framework:

$$
\begin{gathered}
H_{M}=\left\{v=\left(v_{3}, r_{1}, r_{2}\right) \in H^{1}(\omega) \times\left(L^{2}(\omega)\right)^{2}: v_{3}=0 \text { on } \gamma_{0}\right\}, \\
W_{M}=\left\{v=\left(v_{3}, r_{1}, r_{2}\right) \in\left(H^{1}(\omega)\right)^{3}: v_{3}=r_{1}=r_{2}=0 \text { on } \gamma_{0}\right\}, \\
K_{M}=\left\{v \in W_{M}: v_{3} \leq 0 \text { in } \omega_{c}\right\},
\end{gathered}
$$

$\omega_{c}$ being the contact region. The Sobolev spaces $W_{M}$ and $H_{M}$ are respectively equipped with the following norms:

$$
\|v\|_{W_{M}}=\left(\left\|v_{3}\right\|_{1, \omega}^{2}+\left\|r_{1}\right\|_{1, \omega}^{2}+\left\|r_{2}\right\|_{1, \omega}^{2}\right)^{\frac{1}{2}},\|v\|_{H_{M}}=\left(\left\|v_{3}\right\|_{1, \omega}^{2}+\left\|r_{1}\right\|_{0, \omega}^{2}+\left\|r_{2}\right\|_{0, \omega}^{2}\right)^{\frac{1}{2}} .
$$


Let now $a_{M}$ and $b_{M}\left(n^{g}\right)$ be the following positive bilinear forms defined in the spaces $W_{M}$ and $H_{M}$ respectively by:

$$
\begin{gathered}
a_{M}(u, v)=\sum_{\alpha, \beta, \nu, \mu=1}^{2} \int_{\omega} D_{\alpha \beta \nu \mu} \frac{\partial \theta_{\alpha}}{\partial x_{\beta}} \frac{\partial r_{\nu}}{\partial x_{\mu}} d \omega+\sum_{\alpha, \beta=1}^{2} \int_{\omega} G_{\alpha \beta}\left(\theta_{\alpha}+\frac{\partial w_{3}}{\partial x_{\alpha}}\right)\left(r_{\beta}+\frac{\partial v_{3}}{\partial x_{\beta}}\right) d \omega, \\
b_{M}\left(n^{g}\right)(u, v)=\frac{1}{2} \sum_{\alpha, \beta=1}^{2} \int_{\omega} n_{\alpha \beta}^{g} \frac{\partial w_{3}}{\partial x_{\alpha}} \frac{\partial v_{3}}{\partial x_{\beta}} d \omega+\frac{1}{2} \sum_{\alpha, \beta=1}^{2} \int_{\omega} n_{\alpha \beta}^{g} \theta_{\alpha} r_{\beta} d \omega,
\end{gathered}
$$

Where $\left(G_{\alpha \beta}\right)$ denotes the transverse shear rigidity tensor. It is also supposed to verify the same ellipticity property as the tensor $\left(n_{\alpha \beta}^{g}\right)$ [14]. The bilinear forms $a_{M}$ and $b_{M}\left(n^{g}\right)$ are obviously continuous (with constants $M_{M}$ and $N\left(n^{g}\right) / 2$ ) in the spaces $W_{M}$ and $H_{M}$ respectively. Then, following [14], the mapping

$$
|\cdot|_{b_{M}}: H_{M} \rightarrow I R_{+}: v \rightarrow|v|_{b_{M}}=\sqrt{b_{M}\left(n^{g}\right)(v, v)}
$$

is a norm equivalent to the norm $\|\cdot\|_{H_{M}}$ (Raviart et al., 1983), and consequently the embedding of the space $\left(W_{M},\|\cdot\|_{W_{M}}\right)$ in the space $\left(H_{M},|\cdot|_{b_{M}}\right)$ is continuous (with constant $\sqrt{N\left(n^{g}\right) / 2}$ ). Finally, the bilinear form $a_{M}$ is $W_{M}$-elliptic (with constant $\alpha_{M}$ ), see (Ciarlet et al., 1977; Destuynder, 1990 and Duvaut et al., 1972).

Within the framework defined above, the unilateral buckling problem for the Mindlin theory is

$$
a_{M}\left(u_{M}, v-u_{M}\right) \geq \lambda_{M 1} b_{M}\left(n^{g}\right)\left(u_{M}, v-u_{M}\right) \forall v \in K_{M},
$$

where the critical load is solution to the minimizing problem:

$$
\lambda_{M 1}=\min _{v \in K_{M}-\{0\}} \frac{a_{M}(v, v)}{b_{M}\left(n^{g}\right)(v, v)} .
$$

Problem [18] - [19] is mathematically well posed by virtue of theorem 3.1. Denote by $w=\left(w_{3}, \theta_{1}, \theta_{2}\right)$ its solution.

Because the Mindlin's plate model only involves first order partial derivatives, a continuous finite elements scheme of degree 1 is used. This is the triangle with three 
nodes so that the inclusion $K_{M h} \subset K_{M}$ holds. Let $\left(\mathscr{J}_{h}\right)$ be the same regular triangulation as that used for the approximation of the pure membrane problem [8]. Then, the space $W_{M}$ and the convex set $K_{M}$ are respectively approximated by

$$
W_{M h}=\left\{\left(v_{3 h}, r_{1 h}, r_{2 h}\right) \in\left(C^{0}(\bar{\omega})\right)^{3}: v_{3 h \mid T}, r_{1 h \mid T}, r_{2 h \mid T} \in \mathscr{P}_{1}(T), T \in\left(\mathscr{d}_{h}\right) \text { and } v_{3 h}=r_{1 h}=r_{2 h}=0 \text { on } \gamma_{0}\right\} \text {, }
$$

and by

$$
K_{M h}=\left\{\left(v_{3 h}, r_{1 h}, r_{2 h}\right) \in W_{M h}: v_{3 h}\left(x_{i}, y_{j}\right) \leq 0, \text { for all mesh node }\left(x_{i}, y_{j}\right) \in \bar{\omega}_{c}\right\} .
$$

It is obvious that the approximated unilateral buckling problem,

$$
\lambda_{M 1 h}=\min _{v_{h} \in K_{M h}-\{0\}} \frac{a_{M}\left(v_{h}, v_{h}\right)}{b_{M}\left(n^{g}\right)\left(v_{h}, v_{h}\right)},
$$

is well posed (the minimum of a continuous cost over a compact set). Denote by $w_{h}=\left(w_{3 h}, \theta_{1 h}, \theta_{2 h}\right)$ its solution.

The approximated unilateral buckling problem for the Mindlin theory taking into account the error on the membrane efforts tensor will be: find $\lambda_{M 1 h h}>0$ and a nonzero vector $w_{h h}=\left(w_{3 h h}, \theta_{1 h h}, \theta_{2 h h}\right) \in K_{M h}$ such that

$$
\lambda_{M 1 h h}=\min _{v_{h} \in K_{M h}-\{0\}} \frac{a_{M}\left(v_{h}, v_{h}\right)}{b_{M}\left(n_{h}^{g}\right)\left(v_{h}, v_{h}\right)} .
$$

We shall choose

$$
b_{M}\left(n^{g}\right)(w, w)=b_{M}\left(n^{g}\right)\left(w_{h}, w_{h}\right)=b_{M}\left(n_{h}^{g}\right)\left(w_{h h}, w_{h h}\right)=1
$$

so that each of the approximated solutions $w_{h}$ and $w_{h h}$ remains uniformly bounded in $W_{M}$.

First, we are going to establish an error estimate for the unilateral buckling critical load without taking into account the error on the membrane efforts tensor, $\lambda_{M 1 h}-\lambda_{M 1}$, which has already been established in (Ayadi, 2007).

Lemma 5.1. Let $P_{K_{M h}} w$ be the projection, in the sense of the inner product defined by the bilinear form $a_{M}(.,$.$) , of the exact buckling mode w$ on the convex set $K_{M h}$. Then, we have the two following inequalities: 


$$
\left\|w-P_{K_{M h}} w\right\|_{W_{M}} \leq \frac{M_{M}}{\alpha_{M}} \inf _{v_{h} \in K_{M h}}\left\|w-v_{h}\right\|_{W_{M}} .
$$

Furthermore, there exists a positive real $h_{0}$ such that

$$
\left(\lim _{h \rightarrow 0} \inf _{v_{h} \in K_{M h}}\left\|w-v_{h}\right\|_{W_{M}}=0\right) \Rightarrow\left(h \leq h_{0} \Rightarrow \frac{1}{\left|P_{K_{M h}} w\right|_{b_{M}}} \leq 2\right),
$$

Proof: According to the definitions made in lemma 5.1, we have

$$
a_{M}\left(w-P_{K_{M h}} w, v_{h}-P_{K_{M h}} w\right) \leq 0, \forall v_{h} \in K_{M h} .
$$

Using the $W_{M}$-ellipticity and the continuity of the bilinear form $a_{M}$ (with constants $\alpha_{M}$ and $M_{M}$ ) together with [25], for all $v_{h} \in K_{M h}$, we obtain

$$
\begin{aligned}
\alpha_{M}\left\|w-P_{K_{M h}} w\right\|_{W_{M}}^{2} \leq a_{M}\left(w-P_{K_{M h}} w, w-P_{K_{M h}} w\right) \\
\quad=a_{M}\left(w-P_{K_{M h}} w, w-v_{h}\right)+a\left(w-P_{K_{M h}} w, v_{h}-P_{K_{M h}} w\right) \\
\leq a_{M}\left(w-P_{K_{M h}} w, w-v_{h}\right) \\
\quad \leq M_{M}\left\|w-P_{K_{M h}} w\right\|_{W_{M}}\left\|w-v_{h}\right\|_{W_{M}}
\end{aligned}
$$

Hence the inequality [23] is showed.

If $\lim _{h \rightarrow 0} \inf _{v_{h} \in K_{M h}}\left\|w-v_{h}\right\|_{W_{M}}=0$, then, according to [23], the sequence $\left(P_{K_{M h}} w\right)$ converges to $w$ in the space $W_{M}$. Since the embedding of the space $\left(W_{M},\|\cdot\|_{W_{M}}\right)$ in the space $\left(H_{M},|\cdot|_{b_{M}}\right)$ is continuous, the sequence $\left(\left|P_{K_{M h}} w\right|_{b_{M}}\right)$ converges to $|w|_{b_{M}}=1$. Hence the inequality [24] is showed.

Lemma 5.2. Suppose that

$$
\lim _{h \rightarrow 0} \inf _{v_{h} \in K_{M h}}\left\|w-v_{h}\right\|_{W_{M}}=0
$$


Then, the sequence $\left(w_{h}\right)_{h>0}$ is bounded in the norm $\|\cdot\|_{W_{M}}$ :

$$
\left\|w_{h}\right\|_{W_{M}} \leq 2 \sqrt{\frac{M_{M}}{\alpha_{M}}}\|w\|_{W_{M}}
$$

Proof: Using the $W_{M}$-ellipticity of $a_{M}$ and the fact that the projection on a closed convex set is a Lipschitzienne mapping with unit ratio, we obtain

$$
\begin{aligned}
\alpha_{M}\left\|w_{h}\right\|_{W_{M}}^{2} & \leq a_{M}\left(w_{h}, w_{h}\right) \\
& =\inf _{v_{h} \in K_{M h}-\{0\}} \frac{a_{M}\left(v_{h}, v_{h}\right)}{b_{M}\left(n^{g}\right)\left(v_{h}, v_{h}\right)} \\
& \leq \frac{1}{\left|P_{K_{M h}} w\right|_{b_{M}}^{2}} a_{M}\left(P_{K_{M h}} w, P_{K_{M h}} w\right) \\
& \leq \frac{1}{\left|P_{K_{M h}} w\right|_{b_{M}}^{2}} a_{M}(w, w)
\end{aligned}
$$

Then, propriety [24] and the continuity of the bilinear form $a_{M}$ yield [26].

We are now in a position to prove an abstract error estimate for the unilateral buckling critical load of a thin plate, with Mindlin theory and a continuous finite elements scheme, in presence of an obstacle without taking into account the error on the membrane efforts tensor.

Theorem 5.3. Suppose that

$$
\lim _{h \rightarrow 0} \inf _{v_{h} \in K_{M h}}\left\|w-v_{h}\right\|_{W_{M}}=0 .
$$

Then, there exist four positive constants, $c_{1}$ and $c_{2}$ all independent of the subspace $W_{M h}$ and of the convex cone $K_{M h}$ such that

$$
0 \leq \lambda_{M 1 h}-\lambda_{M 1} \leq c_{2} \inf _{v_{h} \in K_{M h}}\left\|w-v_{h}\right\|_{W_{M}}^{2}+c_{1} \inf _{v_{h} \in K_{M h}}\left\|w-v_{h}\right\|_{W_{M}}
$$

Proof: Let $v_{h} \in K_{M h}-\{0\}$, following the expressions of the exact buckling critical load [19] and the approximated one [20], we have 
Error estimates for the buckling load

1023

$$
\begin{aligned}
0 \leq \lambda_{M 1 h}-\lambda_{M 1} & \leq \frac{a_{M}\left(v_{h}, v_{h}\right)}{b_{M}\left(n^{g}\right)\left(v_{h}, v_{h}\right)}-a(w, w) \\
& =a\left(\frac{v_{h}}{\left|v_{h}\right|_{b_{M}}}-w, \frac{v_{h}}{\left|v_{h}\right|_{b_{M}}}-w\right)+2 a\left(w, \frac{v_{h}}{\left|v_{h}\right|_{b_{M}}}-w\right)
\end{aligned}
$$

Hence

$$
\left|\lambda_{M 1 h}-\lambda_{M 1}\right| \leq M_{M}\left\|\frac{v_{h}}{\left|v_{h}\right|_{b_{M}}}-w\right\|_{W_{M}}^{2}+2 M_{M}\|w\|_{W_{M}}\left\|\frac{v_{h}}{\left|v_{h}\right|_{b_{M}}}-w\right\|_{W_{M}}
$$

But the inequality [28] is in particular hold for $v_{h}=P_{K_{M h}} w$.

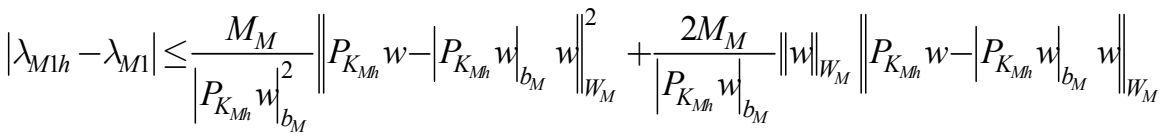

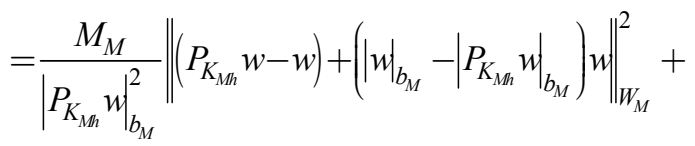

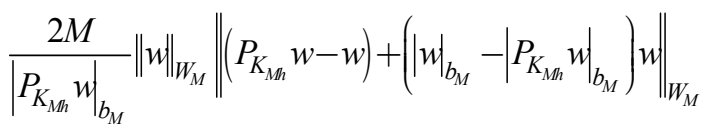

Using the continuity of the embedding of the space $\left(W_{M},\|\cdot\|_{W_{M}}\right)$ in the space

$$
\begin{aligned}
\left(H_{M},|\cdot|_{b_{M}}\right) \text { (with constant } \sqrt{N\left(n^{g}\right) / 2} \text { ), we obtain } \\
\qquad\left|\lambda_{M 1 h}-\lambda_{M 1}\right| \leq \frac{M_{M}}{\left|P_{K_{M h}} w\right|_{b_{M}}^{2}}\left(1+\sqrt{\left.\frac{N\left(n^{g}\right)}{2}\|w\|_{W_{M}}\right)\left\|P_{K_{M h}} w-w\right\|_{W_{M}}^{2}+}\right. \\
\\
\frac{2 M_{M}}{\left|P_{K_{M h}} w\right|_{b_{M}}}\|w\|_{W_{M}}\left(1+\sqrt{\frac{N\left(n^{g}\right)}{2}}\|w\|_{W_{M}}\right)\left\|P_{K_{M h}} w-w\right\|_{W_{M}} .
\end{aligned}
$$


According to [23] and [24], we obtain the inequality [27]: for $h \leq h_{0}$,

$$
\begin{aligned}
\left|\lambda_{M 1 h}-\lambda_{M 1}\right| \leq & \frac{4 M_{M}^{3}}{\alpha_{M}^{2}}\left(1+\sqrt{\frac{N\left(n^{g}\right)}{2}}\|w\|_{W_{M}}\right)^{2} \inf _{v_{h} \in K_{M h}}\left\|w-v_{h}\right\|_{W_{M}}^{2}+ \\
& \frac{4 M_{M}^{2}}{\alpha_{M}}\|w\|_{W_{M}}\left(1+\sqrt{\frac{N\left(n^{g}\right)}{2}}\|w\|_{W_{M}}\right) \inf _{v_{h} \in K_{h}}\left\|w-v_{h}\right\|_{W_{M}} .
\end{aligned}
$$

Second, we are going to estimate the consistency error $\lambda_{M 1 h h}-\lambda_{M 1 h}$.

Lemma 5.4. Suppose that

$$
u^{g} \in\left(H^{k+1}(\omega)\right)^{2} \text { and } \lim _{h \rightarrow 0} \inf _{v_{h} \in K_{M h}}\left\|w-v_{h}\right\|_{W_{M}}=0 .
$$

Then, there exists a positive real $h_{1}$ such that, for all $h \leq h_{1}$, we have:

$$
\frac{1}{b_{M}\left(n_{h}^{g}\right)\left(w_{h}, w_{h}\right)} \leq 4,
$$

the sequence $\left(w_{h h}\right)$ bounded in the norm \|\|$_{W_{M}}$ :

$$
\left\|w_{h h}\right\|_{W_{M}} \leq \frac{4 M_{M}}{\alpha_{M}}\|w\|_{W_{M}},
$$

and consequently

$$
\frac{1}{b_{M}\left(n^{g}\right)\left(w_{h h}, w_{h h}\right)} \leq 4 .
$$

\section{Proof: Let}

$$
W_{M}^{2}=\left\{v=\left(v_{3}, r_{1}, r_{2}\right) \in W_{M}: v_{3} \in H^{2}(\omega)\right\}
$$

be the subspace of $W_{M}$ equipped with the following norm:

$$
\forall v \in W_{M}^{2},\|v\|_{W_{M}^{2}}=\left(\left\|v_{3}\right\|_{2, \omega}^{2}+\left\|r_{1}\right\|_{1, \omega}^{2}+\left\|r_{2}\right\|_{1, \omega}^{2}\right)^{\frac{1}{2}} .
$$


Being inspired by corollary 3.3 , we can easily show that

$$
\left|b_{M}\left(n_{h}^{g}\right)(v, v)-b_{M}\left(n^{g}\right)(v, v)\right| \leq \frac{1}{2} c_{3}(\omega) h^{k}\left|u^{g}\right|_{2, \omega}\|v\|_{W_{M}^{2}}^{2}, \forall v \in W_{M}^{2} .
$$

But we have $\left\|w_{h}\right\|_{W_{M}^{2}}=\left\|w_{h}\right\|_{W_{M}}$ and $\left\|w_{h h}\right\|_{W_{M}^{2}}=\left\|w_{h h}\right\|_{W_{M}}$ since $w_{h}$ and $w_{h h}$ are polynomial functions of degree one by triangle. According to [22], [26] and inequality [32], there exists a positive real $h_{1} \leq h_{0}$ such that, for all $h \leq h_{1}$, we obtain the inequality [29]. Then, using the $W_{M}$-ellipticity of the bilinear form $a_{M}$, we obtain

$$
\begin{aligned}
\alpha_{M}\left\|w_{h h}\right\|_{W_{M}}^{2} & \leq a_{M}\left(w_{h h}, w_{h h}\right) \\
& =\inf _{v_{h} \in K_{M h}-\{0\}} \frac{a_{M}\left(v_{h}, v_{h}\right)}{b_{M}\left(n_{h}^{g}\right)\left(v_{h}, v_{h}\right)} \\
& \leq \frac{a_{M}\left(w_{h}, w_{h}\right)}{b_{M}\left(n_{h}^{g}\right)\left(w_{h}, w_{h}\right)}
\end{aligned}
$$

This, following [29], the continuity of $a_{M}$ and [26], yields [30]. Finally, [32], [22], and [30] involve [31].

We are now in a position to estimate the consistency error $\lambda_{M 1 h h}-\lambda_{M 1 h}$.

Theorem 5.5. Suppose that

$$
u^{g} \in\left(H^{k+1}(\omega)\right)^{2} \text { and } \lim _{h \rightarrow 0} \inf _{v_{h} \in K_{M h}}\left\|w-v_{h}\right\|_{W_{M}}=0 .
$$

Then, for all $h \leq h_{1}$, we have

$$
-\frac{512 M_{M}^{5}}{\alpha_{M}^{4}} c_{3}(\omega)\left|u^{g}\right|_{k+1, \omega}\|n\|_{W_{M}}^{4} h^{k} \leq \lambda_{M 1 h h}-\lambda_{M 1 h} \leq \frac{32 M_{M}^{3}}{\alpha_{M}^{2}} c_{3}(\omega)\left|u^{g}\right|_{k+1, \omega}\|w\|_{W_{M}}^{4} h^{k} \text {.[33] }
$$

Proof: First, we show the right inequality of [33]. Since $w_{h} \in K_{M h}-\{0\}$, [32] yields: 


$$
\begin{aligned}
\lambda_{M 1 h h}-\lambda_{M 1 h} & =\min _{v_{h} \in K_{M h}-\{0\}} \frac{a_{M}\left(v_{h}, v_{h}\right)}{b_{M}\left(n_{h}^{g}\right)\left(v_{h}, v_{h}\right)}-\frac{a_{M}\left(w_{h}, w_{h}\right)}{b_{M}\left(n^{g}\right)\left(w_{h}, w_{h}\right)} \\
& \leq \frac{a_{M}\left(w_{h}, w_{h}\right)}{b_{M}\left(n_{h}^{g}\right)\left(w_{h}, w_{h}\right)}-\frac{a_{M}\left(w_{h}, w_{h}\right)}{b_{M}\left(n^{g}\right)\left(w_{h}, w_{h}\right)} \\
& =\frac{a_{M}\left(w_{h}, w_{h}\right)}{b_{M}\left(n_{h}^{g}\right)\left(w_{h}, w_{h}\right)}\left(b_{M}\left(n^{g}\right)\left(w_{h}, w_{h}\right)-b_{M}\left(n_{h}^{g}\right)\left(w_{h}, w_{h}\right)\right) \\
& \leq \frac{a_{M}\left(w_{h}, w_{h}\right)}{2 b_{M}\left(n_{h}^{g}\right)\left(w_{h}, w_{h}\right)} c_{3}(\omega) h^{k}\left|u^{g}\right|_{k+1, \omega}\left\|w_{h}\right\|_{W_{M}}^{2} .
\end{aligned}
$$

The right inequality of [33] is then showed, for $h \leq h_{1}$, by virtue of the continuity of the bilinear form $a_{M}$ together with [29] and [26].

Second, we show the left inequality of [33]. Since $w_{h h} \in K_{M h}-\{0\}$, [32] yields:

$$
\begin{aligned}
\lambda_{M 1 h}-\lambda_{M 1 h h} & =\min _{v_{h} \in K_{M h}-\{0\}} \frac{a_{M}\left(v_{h}, v_{h}\right)}{b_{M}\left(n^{g}\right)\left(v_{h}, v_{h}\right)}-\frac{a_{M}\left(w_{h h}, w_{h h}\right)}{b_{M}\left(n_{h}^{g}\right)\left(w_{h h}, w_{h h}\right)} \\
& \leq \frac{a_{M}\left(w_{h h}, w_{h h}\right)}{b_{M}\left(n^{g}\right)\left(w_{h h}, w_{h h}\right)}-\frac{a_{M}\left(w_{h h}, w_{h h}\right)}{b_{M}\left(n_{h}^{g}\right)\left(w_{h h}, w_{h h}\right)} \\
& =\frac{a_{M}\left(w_{h h}, w_{h h}\right)}{b_{M}\left(n^{g}\right)\left(w_{h h}, w_{h h}\right)}\left(b_{M}\left(n_{h}^{g}\right)\left(w_{h h}, w_{h h}\right)-b_{M}\left(n^{g}\right)\left(w_{h h}, w_{h h}\right)\right) \\
& \leq \frac{a_{M}\left(w_{h h}, w_{h h}\right)}{2 b_{M}\left(n^{g}\right)\left(w_{h h}, w_{h h}\right)} c_{3}(\omega) h^{k}\left|u^{g}\right|_{k+1, \omega}\left\|w_{h h}\right\|_{W_{M}}^{2}
\end{aligned}
$$

The left inequality of [33] is then showed, for $h \leq h_{1}$, by virtue of the continuity of the bilinear form $a_{M}$ together with [31] and [30].

According to theorem 4.3 and theorem 4.5, an error estimate for the unilateral buckling critical load of a thin plate, in presence of an obstacle, with Mindlin theory, and taking into account the error on the membrane efforts tensor $n_{h}^{g}$ is hereafter achieved.

Corollary 5.6. Suppose that

$$
u^{g} \in\left(H^{2}(\omega)\right)^{2} \text { and } w=\left(w_{3}, \theta_{1}, \theta_{2}\right) \in\left(H^{2}(\omega)\right)^{3} .
$$


Then, there exist four positive constants $C_{1}, C_{2}, C_{3}$ and $C_{4}$ all independent of the subspace $W_{M h}$ and of the convex cone $K_{M h}$ such that

$$
-C_{4} h \leq \lambda_{M 1 h h}-\lambda_{M 1} \leq\left(C_{1}+C_{3}\right) h+C_{2} h^{2}
$$

If $K_{M}=W_{M}$, the error control [34] reduces to

$$
-C_{4} h \leq \lambda_{M 1 h h}-\lambda_{M 1} \leq C_{3} h+C_{2} h^{2}
$$

Proof: Denote by $\pi_{h} v=\left(\pi_{h} v_{3}, \pi_{h} r_{1}, \pi_{h} r_{2}\right)$ the interpolated of degree one of $v=\left(v_{3}, r_{1}, r_{2}\right)$. If $v$ belongs to $K_{M}$, then $\pi_{h} v$ belongs to $K_{M h}$. Therefore, with a regular triangulation $\left(\mathscr{J}_{h}\right)$, see for instance (Raviart et al., 1983), there exists a positive constant $C_{5}$ such that

$$
\begin{aligned}
& \inf _{v_{h} \in K_{M h}}\left\|w-v_{h}\right\|_{W_{M}} \leq\left\|w-\pi_{h} w\right\|_{W_{M}} \\
& \leq C_{5} h|w|_{2, \omega}
\end{aligned}
$$

Adding [27] and [33], and using [36], we obtain [34] with

$$
\begin{gathered}
C_{1}=\frac{4 M_{M}^{2}}{\alpha_{M}}\|w\|_{W_{M}}\left(1+\sqrt{\frac{N\left(n^{g}\right)}{2}}\|w\|_{W_{M}}\right) C_{5}|w|_{2, \omega}, C_{2}=\frac{4 M_{M}^{3}}{\alpha_{M}^{2}}\left(1+\sqrt{\frac{N\left(n^{g}\right)}{2}}\|w\|_{W_{M}}\right)^{2}\left(C_{5}|w|_{2, \omega}\right)^{2}, \\
C_{3}=\frac{32 M_{M}^{3}}{\alpha_{M}^{2}} c_{3}(\omega)\left|u^{g}\right|_{2, \omega}\|w\|_{W_{M}}^{4}, C_{4}=\frac{512 M_{M}^{5}}{\alpha_{M}^{4}} c_{3}(\omega)\left|u^{g}\right|_{2, \omega}\|w\|_{W_{M}}^{4} .
\end{gathered}
$$

If $K_{M}=W_{M}$ (buckling without obstacle) then, for all $v_{h} \in W_{M h}-\{0\}$, we have

$$
\begin{aligned}
\lambda_{M 1 h}-\lambda_{M 1} & \leq \frac{a_{M}\left(v_{h}, v_{h}\right)}{b_{M}\left(n^{g}\right)\left(v_{h}, v_{h}\right)}-a_{M}(w, w) \\
& =a_{M}\left(\frac{v_{h}}{\left|v_{h}\right|_{b_{M}}}-w, \frac{v_{h}}{\left|v_{h}\right|_{b_{M}}}-w\right)+2 a_{M}\left(w, \frac{v_{h}}{\left|v_{h}\right|_{b_{M}}}-w\right)
\end{aligned}
$$

But the last term is negative. Indeed, we have

$$
a_{M}\left(w, \frac{v_{h}}{\left|v_{h}\right|_{b_{M}}}-w\right)=\lambda_{M 1} b_{M}\left(n^{g}\right)\left(w, \frac{v_{h}}{\left|v_{h}\right|_{b_{M}}}-w\right) .
$$


Then, by virtue of the Cauchy Schwartz inequality together with $b_{M}\left(n^{g}\right)(w, w)=1$, we obtain

$$
\begin{aligned}
b_{M}\left(n^{g}\right)\left(w, \frac{v_{h}}{\left|v_{h}\right|_{b_{M}}}-w\right) & \leq\left(\sqrt{b_{M}\left(n^{g}\right)(w, w)} \sqrt{b_{M}\left(n^{g}\right)\left(\frac{v_{h}}{\left|v_{h}\right|_{b_{M}}}, \frac{v_{h}}{\left|v_{h}\right|_{b_{M}}}\right)}-1\right) \\
& =0 .
\end{aligned}
$$

Consequently, the term $C_{1} h$ vanishes in the expression [34], which reduces to [35].

\subsection{Numerical results}

In order to check our theoretical error estimate for the unilateral buckling critical load of a thin plate with Mindlin theory, in presence of an obstacle and taking into account the error on the membrane efforts tensor, the following numerical experiment is handled.

The plate, occupying the two-dimensional domain $\omega=]-0.1,0.1[\times]-0.05,0.05[$, is simply supported on the whole of its edge, clamped on the edge part $\gamma_{0}$ and compressed on the edge part $\gamma_{1}$ by a uniform load $2 \varepsilon \lambda$. Also, the membrane displacements are imposed null on the part edge $\gamma_{0}$ so that the membrane efforts are not trivial and must first be approximated by finite elements scheme. Furthermore, the plate is supposed to have a thickness $2 \varepsilon=0.006 \mathrm{~m}$ and to be made of an elastic, homogenous and isotropic material whose mechanical features are: the Young's modulus $E=1.000 \mathrm{e}+09 P a$, and the Poisson's ratio $\nu=0.3$, see Figure 14 .

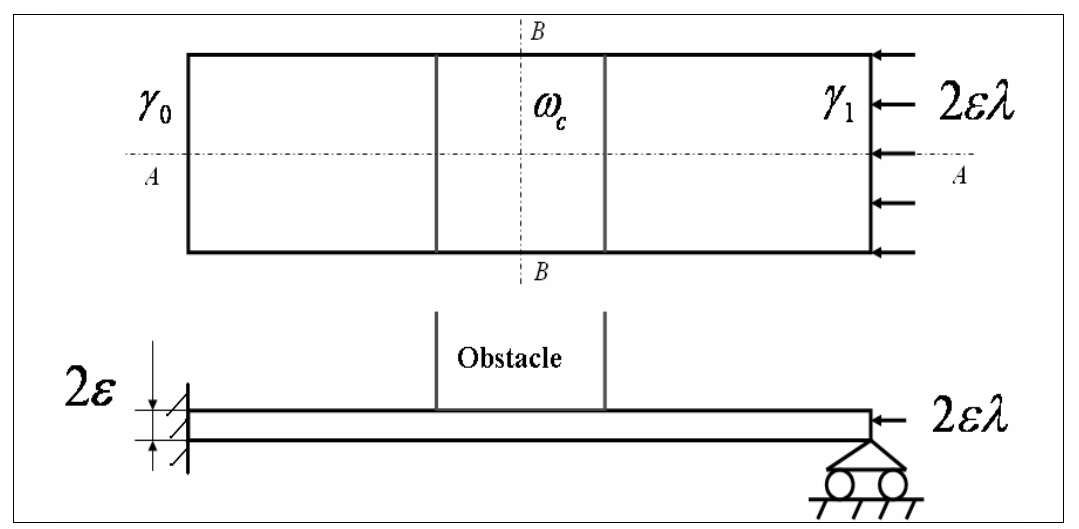

Figure 14. The rectangular plate is in presence of the obstacle 


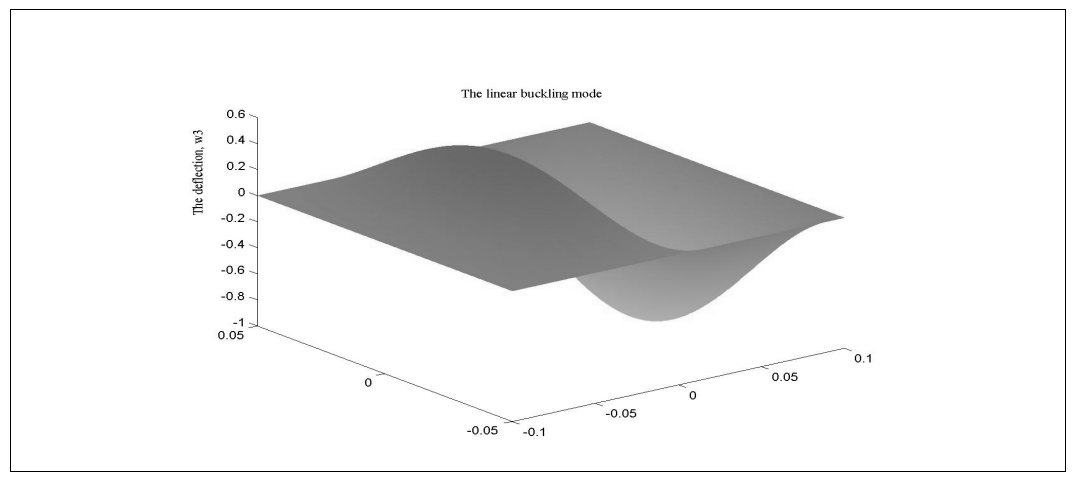

Figure 15. The buckling mode of the plate in the absence of the obstacle

Consider now the obstacle occupying the domain $\bar{\omega}_{c}=[-0.04,0.04] \times[-0.02,0.02]$.

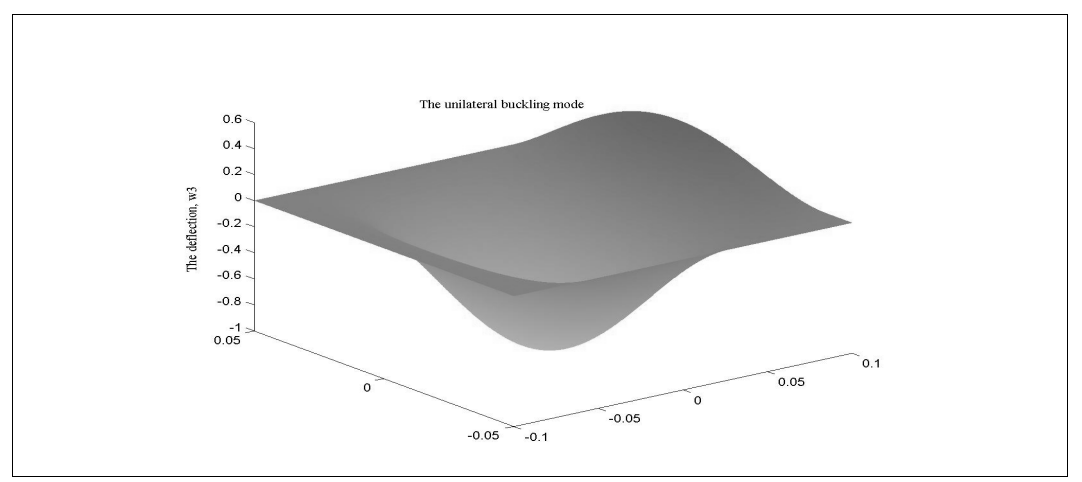

Figure 16. The unilateral buckling mode of the plate

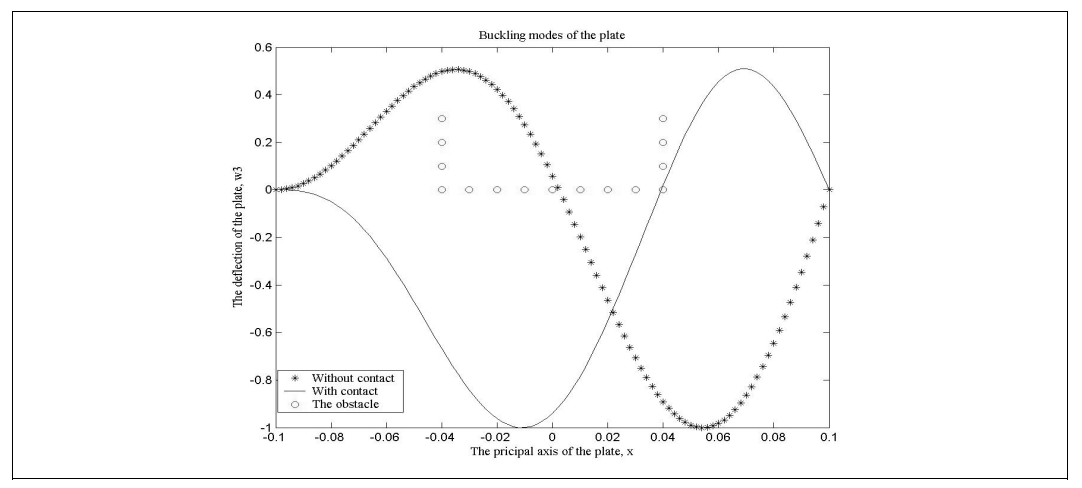

Figure 17. The curves above show the sections $A-A$ of the buckling modes of the plate as well as the obstacle involving the contact domain $\bar{\omega}_{c}=[-0.04,0.04] \times[-0.02,0.02]$ 


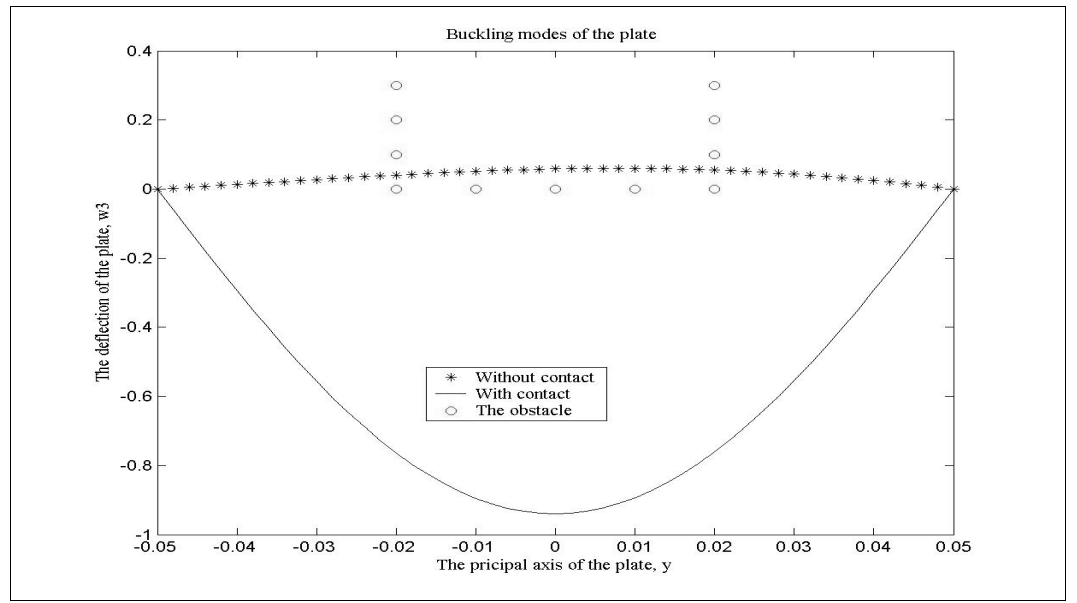

Figure 18. The curves above show the sections $B-B$ of the buckling modes of the plate as well as the obstacle involving the contact domain $\omega_{c}=[-0.04,0.04] \times[-0.02,0.02]$

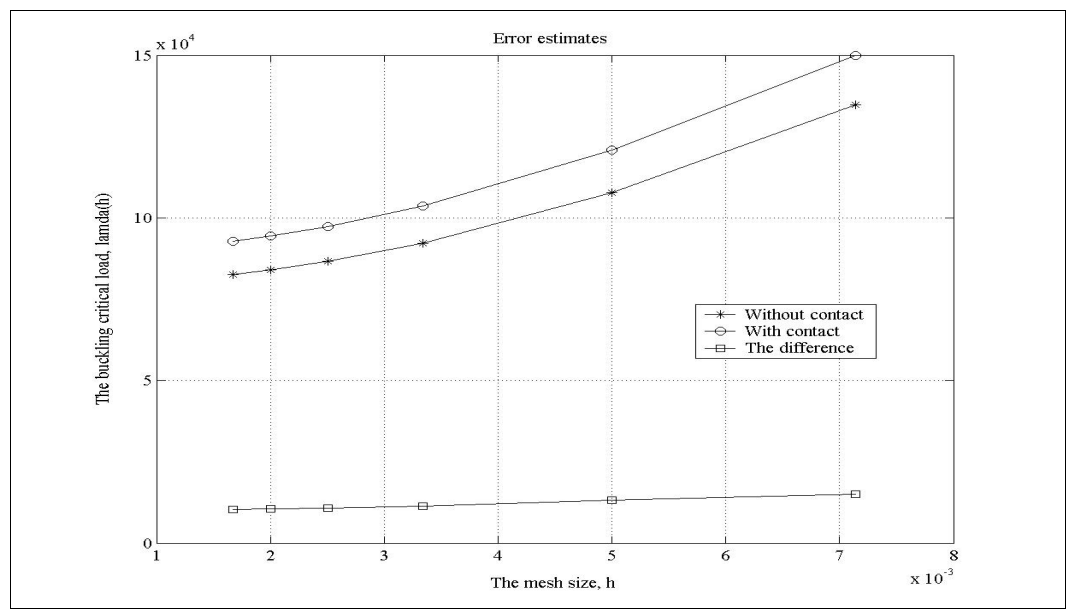

Figure 19. The dependence of the critical load upon the mesh size

The two first curves above give the dependence of the buckling critical load, in the presence and in the absence of the obstacle respectively, upon the mesh size. Following theoretical error estimate formulae [34] and [35], the difference between the two critical loads (with and without contact) must be linear as a function of the mesh size. The third curve shows that this linear behavior is numerically confirmed. 


\section{Kirchhoff-Love's plate model}

We shall approximate the "nonlinear eigenvalue problem" [6] - [7] by using a conformal finite elements method and the same $\operatorname{mesh}\left(\mathscr{J}_{h}\right)$ which was used for approximating the pure membrane problem [8]. Let then $W_{h}$ be a finite-dimensional subspace of the space $W$ such that $W_{h} \subset W$, and $K_{h}$ be a non empty closed convex subset of $W_{h}$ which is a cone. Observe that in general the set $K_{h}$ is not a subset of $K$.

The discrete nonlinear problem, supposed to approach the continuous one [6] [7] without taking into account the error on the membrane efforts tensor (Ayadi, 2007), consists in finding $\left(\lambda_{1 h}, w_{3 h}\right) \in I R_{+}^{*} \times\left(K_{h}-\{0\}\right)$ such that

$$
a\left(w_{3 h}, v_{h}-w_{3 h}\right) \geq \lambda_{1 h} b\left(n^{g}\right)\left(w_{3 h}, v_{h}-w_{3 h}\right), \forall v_{h} \in K_{h},
$$

and

$$
\lambda_{1 h}=\min _{v_{h} \in K_{h}-\{0\}} \frac{a\left(v_{h}, v_{h}\right)}{b\left(n^{g}\right)\left(v_{h}, v_{h}\right)} .
$$

The minimizing problem [38], which involves [37], is mathematically well posed: there exists a function $w_{3 h} \in K_{h}-\{0\}$ which realizes the minimum of problem [38].

The discrete nonlinear problem, supposed to approach the continuous one [6] [7] and taking into account the error on the membrane efforts tensor, consists in finding $\left(\lambda_{1 h h}, w_{3 h h}\right) \in I R_{+}^{*} \times\left(K_{h}-\{0\}\right)$ such that

$$
a\left(w_{3 h h}, v_{h}-w_{3 h h}\right) \geq \lambda_{1 h h} b\left(n_{h}^{g}\right)\left(w_{3 h h}, v_{h}-w_{3 h h}\right), \forall v_{h} \in K_{h},
$$

and

$$
\lambda_{1 h h}=\min _{v_{h} \in K_{h}-\{0\}} \frac{a\left(v_{h}, v_{h}\right)}{b\left(n_{h}^{g}\right)\left(v_{h}, v_{h}\right)} .
$$

From now on, we shall choose

$$
b\left(n^{g}\right)\left(w_{3}, w_{3}\right)=b\left(n^{g}\right)\left(w_{3 h}, w_{3 h}\right)=b\left(n_{h}^{g}\right)\left(w_{3 h h}, w_{3 h h}\right)=1
$$

so that the approximated solutions $w_{3 h}$ and $w_{3 h h}$ remain uniformly bounded in $W$.

An abstract error estimate for the unilateral buckling critical load, $\lambda_{1 h}-\lambda_{1}$, of a thin plate in presence of an obstacle, with Kirchhoff-Love theory and a conformal finite elements scheme $\left(W_{h} \subset W\right)$, without taking into account the error on the 
membrane efforts tensor, has already been established in (Ayadi, 2007). The following theorem recalls it.

Theorem 6.1. Suppose that

$$
\lim _{h \rightarrow 0} \inf _{v_{h} \in K_{h}}\left\|w_{3}-v_{h}\right\|_{2, \omega}=\liminf _{h \rightarrow 0}\left\|w_{3 h}-v\right\|_{2, \omega}=0
$$

Then, there exist four positive constants, $C_{6}, C_{7}, C_{8}$ and $C_{9}$ all independent of the subspace $W_{h}$ and of the convex cone $K_{h}$ such that

$$
-C_{8} \inf _{v \in K}\left\|w_{3 h}-v\right\|_{2, \omega}-C_{9} \inf _{v \in K}\left\|w_{3 h}-v\right\|_{2, \omega}^{2} \leq \lambda_{h}-\lambda_{1} \leq C_{7} \inf _{v_{h} \in K_{h}}\left\|w_{3}-v_{h}\right\|_{2, \omega}^{2}+C_{6} \inf _{v_{h} \in K_{h}}\left\|w_{3}-v_{h}\right\|_{2, \omega}
$$

Proof: See (Ayadi, 2007) where the positive constants are defined by:

$$
\begin{gathered}
C_{6}=\frac{4 M^{2}}{\alpha}\left\|w_{3}\right\|_{2, \omega}\left(1+\sqrt{N\left(n^{g}\right)}\left\|w_{3}\right\|_{2, \omega}\right), C_{7}=\frac{4 M^{3}}{\alpha^{2}}\left(1+\sqrt{N\left(n^{g}\right)}\left\|w_{3}\right\|_{2, \omega}\right)^{2}, \\
C_{8}=\frac{8 M^{\frac{5}{2}}}{\alpha^{\frac{3}{2}}}\left\|w_{3}\right\|_{2, \omega}\left(1+2 \sqrt{\frac{N\left(n^{g}\right) M}{\alpha}}\left\|w_{3}\right\|_{2, \omega}\right), C_{9}=\frac{4 M^{3}}{\alpha^{2}}\left(1+2 \sqrt{\frac{N\left(n^{g}\right) M}{\alpha}}\left\|w_{3}\right\|_{2, \omega}\right)^{2} .
\end{gathered}
$$

We are now going to estimate the consistency error $\lambda_{1 h h}-\lambda_{1 h}$.

Lemma 6.2. Suppose that

$$
u^{g} \in\left(H^{k+1}(\omega)\right)^{2} \text { and } \lim _{h \rightarrow 0} \inf _{v_{h} \in K_{h}}\left\|w_{3}-v_{h}\right\|_{2, \omega}=0 .
$$

Then, there exists a positive real $h_{2}$ such that, for all $h \leq h_{2}$, we have:

$$
\frac{1}{b\left(n_{h}^{g}\right)\left(w_{3 h}, w_{3 h}\right)} \leq 4,
$$

the sequence $\left(w_{3 h h}\right)$ is bounded in the norm $\|\cdot\|_{2, \omega}$ :

$$
\left\|w_{3 h h}\right\|_{2, \omega} \leq \frac{4 M}{\alpha}\left\|w_{3}\right\|_{2, \omega},
$$


and consequently

$$
\frac{1}{b\left(n^{g}\right)\left(w_{3 h h}, w_{3 h h}\right)} \leq 4
$$

Proof: By inspiring of lemma 5.1, we first show that the sequence $\left(w_{3 h}\right)$ is bounded in the norm $\|\cdot\|_{2, \omega}$. Then, by virtue of the inequality [12] in corollary 4.3 and [41], the proof of [43] - [45] is exactly the same as in lemma 5.2 and lemma 5.4.

We are now in a position to estimate the consistency error $\lambda_{1 h h}-\lambda_{1 h}$.

Theorem 6.3. Suppose that

$$
u^{g} \in\left(H^{k+1}(\omega)\right)^{2} \text { and } \lim _{h \rightarrow 0} \inf _{h} \in K_{h}\left\|w_{3}-v_{h}\right\|_{2, \omega}=0 .
$$

Then, for all $h \leq h_{2}$, we have

$$
-\frac{1024 M^{5}}{\alpha^{4}} c_{3}(\omega)\left|u^{g}\right|_{k+1, \omega}\left\|w_{3}\right\|_{2, \omega}^{4} h^{k} \leq \lambda_{1 h h}-\lambda_{1 h} \leq \frac{64 M^{3}}{\alpha^{2}} c_{3}(\omega)\left|u^{g}\right|_{k+1, \omega}\left\|w_{3}\right\|_{2, \omega}^{4} h^{k} .[46]
$$

Proof: The proof of [46], which is a direct consequence of lemma 6.2, is exactly the same as in theorem 5.5.

Finally, according to theorem 6.1 and theorem 6.3 , an abstract error estimate for the unilateral buckling critical load of a thin plate in presence of an obstacle, with Kirchhoff-Love theory, and taking into account the error on the membrane efforts tensor $n_{h}^{g}$ is hereafter achieved.

Corollary 6.4. Suppose that

$$
u^{g} \in\left(H^{k+1}(\omega)\right)^{2} \text { and } \lim _{h \rightarrow 0} \inf _{v_{h} \in K_{h}}\left\|w_{3}-v_{h}\right\|_{2, \omega}=\liminf _{h \rightarrow 0}\left\|w_{3 h}-v\right\|_{2, \omega}=0 .
$$

Then, for all $h \leq h_{2}$, we have

$$
\begin{aligned}
\lambda_{1 h h}-\lambda_{1} \leq & \frac{4 M^{3}}{\alpha^{2}}\left(1+\sqrt{N\left(n^{g}\right)}\left\|w_{3}\right\|_{2, \omega}\right)^{2} \inf _{v_{h} \in K_{h}}\left\|w_{3}-v_{h}\right\|_{2, \omega}^{2}+ \\
& \frac{4 M^{2}}{\alpha}\left\|w_{3}\right\|_{2, \omega}\left(1+\sqrt{N\left(n^{g}\right)}\left\|w_{3}\right\|_{2, \omega}\right) \inf _{v_{h} \in K_{h}}\left\|w_{3}-v_{h}\right\|_{2, \omega}+ \\
& \frac{64 M^{3}}{\alpha^{2}} c_{3}(\omega)\left|u^{g}\right|_{k+1, \omega}\left\|w_{3}\right\|_{2, \omega}^{4} h^{k}
\end{aligned}
$$


and

$$
\begin{aligned}
\lambda_{1 h h}-\lambda_{1} \geq & \frac{-4 M^{3}}{\alpha^{2}}\left(1+2 \sqrt{\frac{N\left(n^{g}\right) M}{\alpha}}\left\|w_{3}\right\|_{2, \omega}\right)^{2} \inf _{v \in K}\left\|w_{3 h}-v\right\|_{2, \omega}^{2}+ \\
& \frac{-8 M^{\frac{5}{2}}}{\alpha^{\frac{3}{2}}}\left\|w_{3}\right\|_{2, \omega}\left(1+2 \sqrt{\frac{N\left(n^{g}\right) M}{\alpha}}\left\|w_{3}\right\|_{2, \omega}\right) \inf _{v \in K}\left\|w_{3 h}-v\right\|_{2, \omega}+ \\
& \frac{-1024 M^{5}}{\alpha^{4}} c_{3}(\omega)\left|u^{g}\right|_{k+1, \omega}\left\|w_{3}\right\|_{2, \omega}^{4} h^{k}
\end{aligned}
$$

REMARK. - Several comments are in order about the abstract error estimate [47][48].

(i) In the linear case, i.e. buckling without obstacle, as $K=W$ and $K_{h}=W_{h} \subset W$, we can easily deduce that

$$
\inf _{w \in K}\left\|w_{3 h}-w\right\|_{2, \omega}=0 \text {, and } a\left(w_{3}, \frac{w_{h}}{\left|w_{h}\right|_{b}}-w_{3}\right) \leq 0, \forall w_{h} \in W_{h} .
$$

The error estimate for the buckling critical load [47] - [48] reduces to

$$
\begin{gathered}
\frac{1024 M^{5}}{\alpha^{4}} c_{3}(\omega)\left|u^{g}\right|_{k+1, \omega}\left\|w_{3}\right\|_{2, \omega}^{4} h^{k} \leq \lambda_{h h}-\lambda_{1} \leq \\
\frac{64 M^{3}}{\alpha^{2}}\left(1+\sqrt{N\left(n^{g}\right)}\left\|w_{3}\right\|_{2, \omega}\right)^{2} \inf _{w_{h} \in W_{h}}\left\|w_{3}-\left.w_{h}\right|_{2, \omega} ^{2}+\left.u^{g}\right|_{k+1, \omega}\right\| w_{3} \|_{2, \omega}^{4} h^{k}
\end{gathered}
$$

(ii) Observe that the approximation method in the nonlinear case [47] - [48] would be as accurate as that of linear case [49] if we have

$$
\inf _{w \in K}\left\|w_{3 h}-w\right\|_{2, \omega}=O\left(h^{k+\varepsilon}\right) \text { and } \inf _{w \in K}\left\|w_{3 h}-w\right\|_{2, \omega}=O\left(h^{k+\varepsilon}\right), \varepsilon \geq 0 .
$$

(iii) If the inclusion $K_{h} \subset K$ holds, then obviously the term $\inf _{w \in K}\left\|w_{3 h}-w\right\|_{2, \omega}$, which is expected to be the hardest to evaluate (Ciarlet, 1978), vanishes in the error estimate formula [48]. 


$$
\begin{aligned}
\frac{1024 M^{\rho}}{\alpha^{4}} c_{3}(\omega)\left|u^{g}\right|_{k+1, \omega}\left\|w_{3}\right\|_{2, \omega}^{4} h^{k} \leq \lambda_{h h}-\lambda & \leq \frac{4 M^{\beta}}{\alpha^{2}}\left(1+\sqrt{N\left(n^{g}\right)}\left\|w_{3}\right\|_{2, \omega}\right)^{2} \inf _{w_{h} \in K_{h}}\left\|w_{3}-w_{h}\right\|_{2, \omega}^{2}+ \\
& \frac{4 M^{2}}{\alpha}\left\|w_{3}\right\|_{2, \omega}\left(1+\sqrt{N\left(n^{g}\right)}\left\|w_{3}\right\|_{2, \omega}\right)_{w_{h} \in K_{h}}\left\|w_{3}-w_{h}\right\|_{2, \omega} \\
& +\frac{64 M^{3}}{\alpha^{2}} c_{3}(\omega)\left|u^{g}\right|_{k+1, \omega}\|w\|_{2, \omega}^{4} h^{k} .
\end{aligned}
$$

(iv) Following the error estimate [50], the optimal choice of the space $W_{h}$ is such that

$$
\inf _{w_{h} \in K_{h}}\left\|w_{3}-w_{h}\right\|_{2, \omega}=O\left(h^{k}\right)
$$

\section{Conclusion and perspectives}

The Kirchhoff-Love plate theory requires a continuous differentiable finite elements scheme. It is not only expensive and difficult to implement numerically, but also leads to the abstract error estimate [47] - [48] whose term, $\inf _{w \in K}\left\|w_{3 h}-w\right\|_{2, \omega}$, is very hard to evaluate. Using the very interesting result, namely the maximum of two functions in $H^{1}(\omega)$ is also in $H^{1}(\omega)$ (Lewy et al., 1969), Ciarlet has succeeded, in the equilibrium position of an elastic membrane problem in presence of an obstacle, to evaluate the term $\inf _{w \in K}\left\|w_{3 h}-w\right\|_{1, \omega}$ (Ciarlet, 1978). So, if the maximum of two functions in $H^{2}(\omega)$ is also in $H^{2}(\omega)$ (which is not true if $\omega$ is an open subset of $I R)$, then there is a hope to evaluate the term $\inf _{w \in K}\left\|w_{3 h}-w\right\|_{2, \omega}$.

The Mindlin plate theory requires - at the opposite - only continuous finite elements scheme. Using the Mindlin plate model together with a conformal finite elements method of very low degree (linear local interpolation) is sufficient to approach the unilateral buckling critical load of a thin plate in presence of an obstacle, as exemplify both of the theoretical error estimate [34] - [35] and the numerical results presented in Figure 19.

In the future work, we are going to deal with achieving an error estimate for the unilateral critical load using the Mindlin plate theory and an interpolation of degree two (triangle with six nodes). The approximated space becomes

$$
W_{M h}=\left\{\left(v_{3 h}, r_{1 h}, r_{2 h}\right) \in\left(C^{0}(\bar{\omega})\right)^{3}: v_{3 h \mid T}, r_{1 h \mid T}, r_{2 h \mid T} \in \mathcal{P}_{2}(T), T \in\left(\mathscr{E}_{h}\right) \text { and } v_{3 h}=r_{1 h}=r_{2 h}=0 \text { on } \gamma_{0}\right\} \text {. }
$$


In this case, the unilateral contact constraints convex $K$ is approximated by

$$
K_{M h}=\left\{\left(v_{3 h}, r_{1 h}, r_{2 h}\right) \in W_{M h}: v_{3 h}\left(x_{i}, y_{j}\right) \leq 0, \text { for all mesh node }\left(x_{i}, y_{j}\right) \in \bar{\omega}_{c}\right\},
$$

which is not a subset of $K_{M}$. So, we must be able to evaluate the term $\inf _{v \in K}\left\|w_{h}-v\right\|_{W_{M}}$. By virtue of the Lewy-Stampacchia result (Lewy et al., 1969), this task is easier than the evaluation of $\inf _{w \in K}\left\|w_{3 h}-w\right\|_{2, \omega}$ because $W_{M} \subset\left(H^{1}(\omega)\right)^{3}$.

\section{References}

Ayadi M., Nevers T., «Un modèle Eulerien de calcul de la charge critique de flambement d'une plaque mince multicouches délaminée », Les Annales de l'ENIT, vol. 4, n 1, 1990.

Ayadi M., «Sur un algorithme pour rendre compte du contact unilatéral dans une plaque délaminée », Les Annales Maghrébines de l'Ingénieur, vol. 7, n² 2, 1993.

Ayadi M., "An algorithm for computing the critical state of unilateral buckling of thin plates", European Journal of Computational Mechanics, vol. 15, $\mathrm{n}^{\circ}$ 4, 2006, p. 341-358.

Ayadi M., "Error estimates for the unilateral buckling critical load of a thin plate", European Journal of Computational Mechanics, vol. 16, n5, 2007, p. 583-600.

Bradford M.A., Smith S.T., Oehlers D.J., "Semi-Compact steel plates with unilateral restraint subjected to bending, compression and shear", Journal of Constructional Steel Research, vol. 56, 2000, p. 47-67.

Brézis H., Analyse fonctionnelle et applications, Collection Mathématiques Appliquées pour la Maîtrise, Paris, Masson, 1983.

Chai H., "On the post-buckling behavior of bilaterally constrained plates", International Journal of Solids and Structures, vol. 39, $\mathrm{n}^{\circ}$ 11, 2002, p. 2911-2926.

Ciarlet P.G., Elasticité tridimensionnelle, Collection Recherches en Mathématiques Appliquées, Paris, Masson, 1986.

Ciarlet P.G, The Finite Element Method for Elliptic Problems, Series "Studies in Mathematics and its Applications", North-Holland, Amsterdam, 1978.

Ciarlet P.G., Destuynder P., « Une justification du modèle biharmonique en théorie linéaire des plaques », C. R. Acad. Sci. Paris, Sér. A 285, 1977, p. 851-854.

Cimetière A., « Un problème de flambement unilatéral en théorie des plaques », Journal de Mécanique, vol. 19, n 1, 1980, p. 183-202.

Cimetière A., " Méthode de Liapounov-Schmidt et branche de bifurcation pour une classe d'inéquations variationnelles », C. R. Acad. Sci. Paris, t. 300, Sér. I, n 15, 1985, p. 565-568. 
Davet J., Destuynder Ph., « Singularités logarithmiques dans les effets de bord d'une plaque en matériaux composites », Journal de Mécanique Théorique et Appliquée, vol. 4, 1985, p. $357-380$.

Destuynder Ph., Modélisation des coques minces élastiques, Collection Physique Fondamentale et Appliquée, Paris, Masson, 1990.

Do C., «Problèmes de valeurs propres pour une inéquation variationnelle sur un cône et application au flambement unilatéral d'une plaque mince », C. R. Acad. Sci. Paris, Sér. A 280, 1975, p. 45-48.

Do C., "The buckling of a thin elastic plate subjected to unilateral conditions", Applications of Methods of Functional Analysis to Problems in Mechanics, Springer Lecture Notes, $\mathrm{n}^{\circ} 503,1976$, p. 307-316.

Duvaut G., Lions J., Les inéquations en Physique et en Mécanique, Paris, Dunod, 1972.

Febres R., Inglessis P., Florez-Lopez J., "Modeling of local buckling in tubular steel frames subjected to cyclic loading", Computers and Structures, vol. 81, 2003, p. 2237-2247.

Goeleven D., Motreanu D., “Asymptotic Eigenvalues and Spectral Analysis of Variational Inequalities", Communications in Applied Analysis, n 2, 1998, p. 343-372.

Goeleven D., Théra M., "Nonlinear Variational Inequalities Depending on a Parameter", Serdica Mathematical Journal, $\mathrm{n}^{\circ}$ 21, 1995, p. 1001-1017.

Kucera M., "A New Method for Obtaining Eigenvalues of Variational Inequalities: Operators with Multiple Eigenvalues", Czechoslovak Mathematical Journal, n 32, 1982, p. 197-207.

Lewy H., Stampacchia G., "On the regularity of solutions of a variational inequality", Comm. Pure Appl. Math., 22, 1969, p. 153-188.

Ma X., Butterworth J.W., Clifton C., "Compressive buckling analysis of plates in unilateral contact”, International Journal of Solids and Structures, vol. 44, 2007, p. 2852-2862.

Mouradova A.D., Stavroulakis G.E., "A unilateral contact model with buckling in von Karman plates”, Nonlinear Analysis: Real World Applications, Article in press, 2006.

Nevers Th., Modélisation Théorique et Numérique du Délaminage des Plaques Composites, Thèse de l'Ecole Centrale de Paris, 1986.

Parry G., Colin J., Coupeau C., Foucher F., Cimetière A., Grilhé J., "Effect of substrate compliance on the global unilateral post-buckling of coatings: AFM observations and finite element calculations", Acta Materialia, vol. 53, 2005, p. 441-447.

Quittner P., "Spectral Analysis of Variational Inequalities", Commentationes Mathematicae Universitatis Carolinae, $\mathrm{n}^{\circ}$ 27, 1986, p. 605-629.

Raviart P.A., Thomas J.M., Introduction à l'Analyse Numérique des Equations aux Dérivées Partielles, Collection Mathématiques Appliquées pour la Maîtrise, Paris, Masson, 1983.

Riddell R.C., "Eigenvalue Problems for Nonlinear Elliptic Variational Inequalities on a Cone", Journal of Functional Analysis, n² 26, 1977, p. 333-355. 
Shahwan K.W., Waas A.M., "Buckling of unilaterally constrained plates: applications to the study of delaminations in layered structures", Journal of The Franklin Institute, vol. 335, $\mathrm{n}^{\circ} 8,1998$, p. 1009-1039.

Shen H.S., Li Q.S., "Postbuckling of shear deformable laminated plates resting on a tensionless elastic foundation subjected to mechanical or thermal loading", International Journal of Solids and Structures, vol. 41, 2004, p. 4769-4785.

Shiri S., Ayadi M., «Flambement d'une plaque multicouche délaminée. Prise en compte du contact unilatéral », $2^{e}$ Congrès International : Conception et Modélisation des Systèmes Mécaniques, Monastir, Tunisie, 19-21 Mars, 2007.

Smith S.T., Bradford M.A., Oehlers D.J., "Elastic buckling of unilaterally constrained rectangular plates in pure shear", Engineering Structures, vol. 21, n 5, 1999, p. 443-453.

Smith S.T., Bradford M.A., Oehlers D.J., "Unilateral buckling of elastically restrained rectangular mild steel plates", Computational Mechanics, vol. 26, n 4, 2000, p. 317-324.

Article reçu le 5 juillet 2007 Accepté après revisions le 11 juin 2008 